\title{
DETERMINATION OF THERMAL DIFFUSIVITY, CONDUCTIVITY, AND ENERGY RELEASE FROM THE INTERNAL TEMPERATURE PROFILES OF ENERGETIC MATERIALS
}

\author{
W. W. Erikson, M. A. Cooper, M. L. Hobbs, M. J. Kaneshige, M. S. Oliver, and S. Snedigar \\ Sandia National Laboratories* * \\ Albuquerque, NM
}

\begin{abstract}
A novel data processing technique has been developed to obtain thermal diffusivity, conductivity, and reaction heat release for energetic materials from Sandia Instrumented Thermal Ignition (SITI) experiments heated with a linear ramp temperature boundary condition. The method is based on the equivalence of the temperature reponses of: (a) ramped temperature boundary condition with no internal heat generation and (b) uniform heat generation (that is, with a negative value) with constant temperature boundary conditions; which is true regardless of the spatial domain. For the specific case analyzed herein (the SITI apparatus), the midplane temperature profile is well represented by a quadratic expression in the radial coordinate for both ramped boundary temperature and uniform heat generation responses. Internal temperature data from temperature ramped SITI experiments with various pyrotechnics, propellants, and explosives were analyzed. Quadratic fits to the temperature profile data were made and the associated fitting coefficients were converted to yield thermal diffusivity directly. Thermal conductivity was then determined from thermal diffusivity, given knowledge of the material's specific heat capacity and density. Finally, because of the equivalence of the cases (a) and (b) above, their individual contributions to a combined temperature profile can be easily separated, thereby yielding internal heat generation as well. This technique allows for measurements of properties for pressed and powdered materials over a range of densities and temperatures. The technique is demonstrated using pyrotechnic materials $\left(\mathrm{KClO}_{4}\right.$ and $\left.\mathrm{Ti} / \mathrm{KClO}_{4}\right)$, a composite solid propellant (herein referred to as "Propellant A", a class 1.3 AP-HTPB-aluminum propellant) and an explosive (PBX 9502).
\end{abstract}

\section{INTRODUCTION}

The thermal properties of energetic materials play important roles in their behavior under thermal loading. In order to accurately predict behavior of energetic components, devices and systems, it is essential that one have accurate material property values-particularly for properties to which the material behavior is highly sensitive. For example, models of pyrotechnic materials have shown the pyrotechnic thermal conductivity to significantly impact the time to ignition for hot wire devices [1].

There are a variety of techniques which have been used to determine thermal diffusivity and conductivity - a cursory examination of the American Society for Testing and Materials (now ASTM International) website [2] lists dozens of standard procedures for measuring thermal conductivity of materials of various types-some of which would be inappropriate for use with energetic materials. However, our purpose here is not to provide an exhaustive list of available methods and to enumerate their various advantages and disadvantages, but rather to suggest a new way to derive this information from tests on energetic materials conducted with the various versions of the Sandia Instrumented Thermal Ignition (SITI) apparatus - tests which are already being conducted for other reasons. It is expected that by careful analysis of the experiments, additional material property information can be gleaned from these tests.

Other approaches including inverse methods [3] and optimization techniques [4] have been applied to SITI to obtain information on material properties and/or energy release. One difference here is that the approach to be presented does not require the use of a computational model for data processing. Once the initial geometric scaling factor has been established, the data can be processed directly. This work therefore represents a data analysis technique, rather than a modeling and simulation technique.

\footnotetext{
* Sandia National Laboratories is a multi-program laboratory managed and operated by Sandia Corporation, a wholly owned subsidiary of Lockheed Martin Corporation, for the U.S. Department of Energy's National Nuclear Security Administration under contract DE-AC04-94AL85000. This work was performed under internal funding.
} 
The original SITI device [5] has been used to investigate the behavior of over 30 different energetic materials (EMs) including explosives, propellants, and pyrotechnics. In these tests, two 1" (2.54 $\mathrm{cm}$ ) diameter by 0.5 " $(1.27 \mathrm{~cm})$ high cylindrical samples of an EM are enclosed within aluminum confinement. Nine thermocouples (TCs) are laid horizontally in a grid between the two EM samples. Orings maintain a hermetic seal with Kapton ${ }^{\circledR}$ sheets to electrically isolate the TC wires. A feedbackcontrolled rope heater wrapped around the outside surface of the confinement provides heating to the device.

Modifications to SITI were made to accommodate the higher temperatures required to study inorganic pyrotechnic materials. These modifications include the use of a flat gasket made of Garlock $^{\circledR}$ 9900 for sealing, the application of screw-mounted TCs on the exterior, and a simplification of the interior cavity shape (expansion gap was removed). The resulting apparatus became known as High Operating Temperature SITI (HOT-SITI). Figure 1 shows a cutaway illustration of the SITI and HOT-SITI devices.
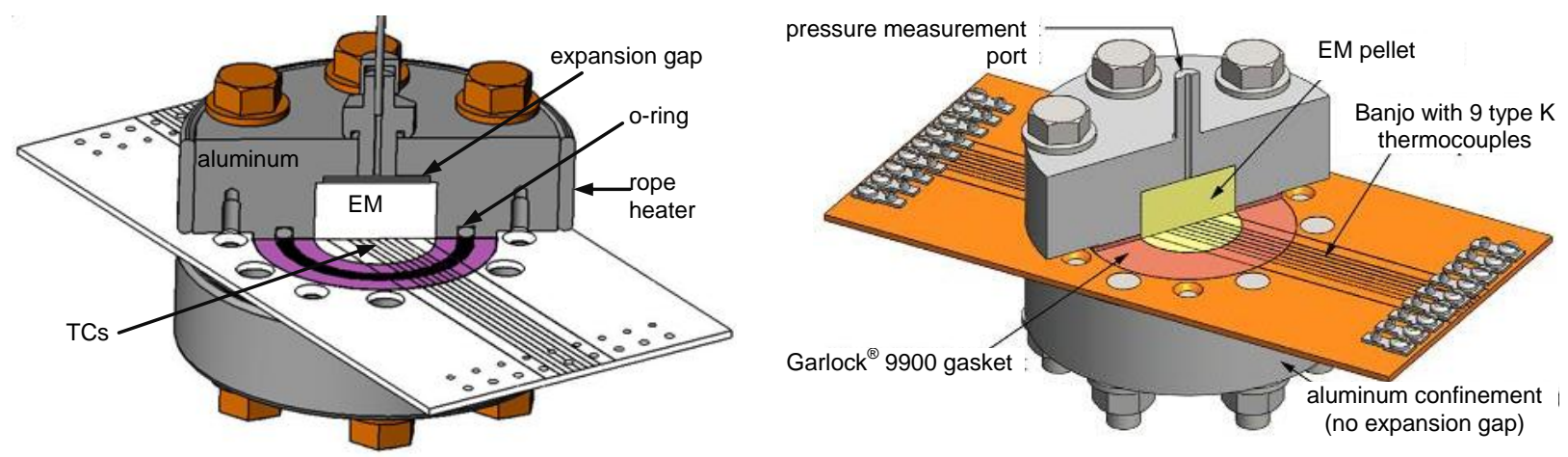

Fig. 1: Cutaway of SITI devices. Left: Original SITI; Right: HOT-SITI.

To date, HOT-SITI has been used with several different pyrotechnics or pyrotechnic ingredients, including $\mathrm{TiH}_{1.65}-\mathrm{KClO}_{4}$ (THKP), Ti-KClO 4 powder (41\% Ti, 59\% $\mathrm{KClO}_{4}$ aka TKP output), and pure $\mathrm{KClO}_{4}$. In all of these tests, the powdered samples were pressed to form monolithic pellets, typically with about 18 to $20 \%$ porosity. Details of tests of the HOT-SITI apparatus are described elsewhere [6, 1]. Here we will demonstrate some analysis techniques using test data for TKP output material and $\mathrm{KClO}_{4}$.

\section{BACKGROUND}

In a fully enclosed device such as HOT-SITI, the temperature profile produced in the interior is determined (if internal self-heating from reactions can be ignored) by heating the exterior. With its high thermal conductivity, the aluminum confinement provides a near isothermal boundary condition. By varying the amount of heat supplied to the aluminum, various temperature histories can be produced. The typical mode of operation in SITI experiments has been a fast $\left(\sim 10^{\circ} \mathrm{C} / \mathrm{min}\right)$ ramp to a set temperature, followed by a constant temperature soak. During the soak period, chemical reactions within the interior of the energetic material proceed and self-heating occurs-eventually leading to a thermal runaway ignition event. The ramp-and-hold profile allows the energy release from reactions to be studied in considerable detail (because the responses of the interior TCs become separated temporally), which has led to improved thermal decomposition reaction models [3].

However, the same ramp-and-hold heating profiles are inadequate for inorganic pyrotechnic materials. Because of the diffusion-limited reaction behavior of two-component pyrotechnics, a rampand-hold profile tends to not produce a full ignition event but rather a thermal excursion which eventually self-quenches as condensed phase reaction products form a passivation barrier between the oxidizer and fuel particles [1, 3]. As a consequence, most of the HOT-SITI tests with pyrotechnic materials have been performed with a temperature ramp profile-temperature is continually increased in a linear fashion until an ignition event occurs. 
During the course of analyzing the temperature profiles for the ramped HOT-SITI tests, it was observed that the spatial variations (i.e. T as a function of $r$ at the device mid-plane) were all very similar in shape-near perfect quadratic profiles in r. While the "depth" of the profile would differ based on heating rate, each produced a quadratic profile (see examples in Fig. 2). It was hoped that this information would be useful to obtain relevant material parameters from the test data. After some careful analysis, we discovered that thermal diffusivity and reaction heat release can be obtained. The process of doing so is the subject of this paper.

\section{MATHEMATICAL BASIS FOR ANALYSIS}

It can be easily demonstrated that the spatial temperature profile produced in an arbitrary-shaped object by a ramped boundary condition and zero volumetric source is exactly the same as the profile produced by a constant, uniform volumetric source term of appropriate value in the same object with constant Dirichlet boundary conditions. While this can be proven in the general case for a linear system, here we focus on a specific geometry of interest-a finite solid cylinder reminiscent of SITI. We obtain analytic solutions for the cylindrical geometry by methods in heat transfer texts $[7,8,9]$.

Here we begin by considering the initial boundary value problem for the temperature in a finite cylinder defined over the radial region $0 \leq r \leq R$ and axial region $-L \leq z \leq L$, as shown in Fig. 3 . The governing equation is defined by Eq. (1) subject to its initial condition, boundary conditions and source term for the cases of interest (Table I). In Eq. (1), $\alpha$ is the thermal diffusivity $\left[\mathrm{m}^{2} / \mathrm{s}\right], \lambda$ is thermal conductivity $[\mathrm{W} / \mathrm{m}-\mathrm{K}]$, and $\mathrm{Q}$ is a volumetric source term $\left[\mathrm{W} / \mathrm{m}^{3}\right]$.

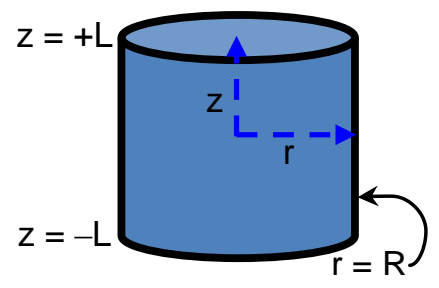

$$
\frac{1}{r} \frac{\partial}{\partial r} r \frac{\partial T}{\partial r}+\frac{\partial^{2} T}{\partial z^{2}}+\frac{Q}{\lambda}=\frac{1}{\alpha} \frac{\partial T}{\partial t}
$$

Fig. 3. Finite cylinder geometry

Table I: Initial and Boundary Conditions for Ramped, Volume Source, and Combined Cases.

\begin{tabular}{|l|c|c|c|}
\hline & (a) Ramp: & (b) Volume Source: & (c) Combined: \\
& Ramp BC, Zero Source & Zero BC, Uniform Source & Ramp BC, Uniform Source \\
\hline Initial Condition & $\mathrm{T}\left(\mathrm{r}, \mathrm{z}, \mathrm{t}_{0}\right)=0$ & $\mathrm{~T}\left(\mathrm{r}, \mathrm{z}, \mathrm{t}_{0}\right)=0$ & $\mathrm{~T}\left(\mathrm{r}, \mathrm{z}, \mathrm{t}_{0}\right)=0$ \\
\hline Boundary & $\mathrm{T}(\mathrm{R}, \mathrm{z}, \mathrm{t})=\mathrm{Ht}$ & $\mathrm{T}(\mathrm{R}, \mathrm{z}, \mathrm{t})=0$ & $\mathrm{~T}(\mathrm{R}, \mathrm{z}, \mathrm{t})=\mathrm{Ht}$ \\
Conditions & $\mathrm{T}(\mathrm{r}, \pm \mathrm{L}, \mathrm{t})=\mathrm{Ht}$ & $\mathrm{T}(\mathrm{r}, \pm \mathrm{t}, \mathrm{t})=0$ & $\mathrm{~T}(\mathrm{r}, \pm \mathrm{t}, \mathrm{t})=\mathrm{Ht}$ \\
\hline Source Term & $\mathrm{Q}=0$ & $\mathrm{Q}=\mathrm{Q}_{0}$ & $\mathrm{Q}=\mathrm{Q}_{0}$ \\
\hline
\end{tabular}

\footnotetext{
* An interesting exercise is to use a finite element code to model an arbitrary geometry initially at $\mathrm{T}=\mathrm{T}_{0}$ in which the entire exterior surface is subjected to a temperature ramp boundary condition rising at heating rate, $\mathrm{H}$ [units of $\mathrm{K} / \mathrm{s}$ ], and the entire interior has a constant volumetric source term of value $\mathrm{Q}=\rho \mathrm{C}_{\mathrm{p}} \mathrm{H}$. For constant density and specific heat, the temperature profile of the entire geometry will remain spatially uniform over time; the temperature at every location precisely given by $\mathrm{T}=\mathrm{T}_{0}+\mathrm{Ht}$. This choice of volumetric heat source exactly counteracts the profile that would normally result from a change in boundary temperature.
} 
The full analytic solution for the profile shape from a temperature ramped finite cylinder (case a) is given here as Eq. (2a). The corresponding solution for the constant source, zero boundary condition (case b) is given in Eq. (2b).

$$
\begin{gathered}
T_{\text {ramp }}(r, z, t)=H t-\frac{32 H R^{2} A^{2}}{\alpha \pi} \sum_{n=0}^{\infty} \sum_{m=1}^{\infty} \frac{(-1)^{n}}{(2 n+1) \beta_{m}\left(4 A^{2} \beta_{m}^{2}+(2 n+1)^{2} \pi^{2}\right)} \frac{J_{0}\left(r \beta_{m} / R\right)}{J_{1}\left(\beta_{m}\right)} \cos \left[\frac{(2 n+1) \pi z}{2 R A}\right] \\
+\frac{32 H R^{2} A^{2}}{\alpha \pi} \sum_{n=0}^{\infty} \sum_{m=1}^{\infty} \frac{(-1)^{n}}{(2 n+1) \beta_{m}\left(4 A^{2} \beta_{m}^{2}+(2 n+1)^{2} \pi^{2}\right)} \frac{J_{0}\left(r \beta_{m} / R\right)}{J_{1}\left(\beta_{m}\right)} \cos \left[\frac{(2 n+1) \pi z}{2 R A}\right] \exp \left[-\frac{\alpha t}{R^{2}}\left(\beta_{m}^{2}+\frac{(2 n+1)^{2} \pi^{2}}{4 A^{2}}\right)\right] \\
T_{\text {volsrc }}(r, z, t)=\frac{32 Q_{o} R^{2} A^{2}}{\lambda \pi} \sum_{n=0}^{\infty} \sum_{m=1}^{\infty} \frac{(2 n+1) \beta_{m}\left(4 A^{2} \beta_{m}^{2}+(2 n+1)^{2} \pi^{2}\right)}{\frac{J_{0}}{J_{1}\left(\beta_{m} / R\right)}} \cos \left[\frac{(2 n+1) \pi z}{2 R A}\right] \\
-\frac{32 Q_{0} R^{2} A^{2}}{\lambda \Pi} \sum_{n=0}^{\infty} \sum_{m=1}^{\infty} \frac{(-1)^{n}}{(2 n+1) \beta_{m}\left(4 A^{2} \beta_{m}^{2}+(2 n+1)^{2} \pi^{2}\right)} \frac{J_{0}\left(r \beta_{m} / R\right)}{J_{1}\left(\beta_{m}\right)} \cos \left[\frac{(2 n+1) \pi z}{2 R A}\right] \exp \left[-\frac{\alpha t}{R^{2}}\left(\beta_{m}^{2}+\frac{(2 n+1)^{2} \pi^{2}}{4 A^{2}}\right)\right]
\end{gathered}
$$

Here $T$ is temperature $[K], r$ and $z$ are spatial variables [m], $t$ is time $[s], \alpha$ is thermal diffusivity $\left[\mathrm{m}^{2} / s\right], H$ is the heating rate $[\mathrm{K} / \mathrm{s}], \mathrm{J}_{0}$ and $\mathrm{J}_{1}$ are Bessel functions of the first kind, $\beta_{\mathrm{m}}$ are positive roots of $\mathrm{J}_{0}(\beta)=0$ (i.e. $\left.\beta_{1}=2.405, \beta_{2}=5.520, \beta_{3}=8.654, \ldots\right)$ and $A$ is the overall aspect ratio of the cylinder $(A=2 L / D=L / R)$. The "ramp" and "volsrc" subscript indicates a temperature ramped or constant source term solution respectively. Note the similarity between the two solutions: all terms in the summations are exactly identical and their associated coefficients are similar as well. This allows a very simple superposition of the two solutions to yield the combined solution (2c):

$$
\begin{aligned}
& T(r, z, t)=H t+\frac{32 R^{2} A^{2}}{\pi}\left(\frac{Q_{0}}{\lambda}-\frac{H}{\alpha}\right)\left\{\sum_{n=0}^{\infty} \sum_{m=1}^{\infty} \frac{(-1)^{n}}{(2 n+1) \beta_{m}\left(4 A^{2} \beta_{m}^{2}+(2 n+1)^{2} \pi^{2}\right)} \frac{J_{0}\left(r \beta_{m} / R\right)}{J_{1}\left(\beta_{m}\right)} \cos \left[\frac{(2 n+1) \pi z}{2 R A}\right]\right. \\
& \left.\quad-\sum_{n=0}^{\infty} \sum_{m=1}^{\infty} \frac{(-1)^{n}}{(2 n+1) \beta_{m}\left(4 A^{2} \beta_{m}^{2}+(2 n+1)^{2} \pi^{2}\right)} \frac{J_{0}\left(r \beta_{m} / R\right)}{J_{1}\left(\beta_{m}\right)} \cos \left[\frac{(2 n+1) \pi z}{2 R A}\right] \exp \left[-\frac{\alpha t}{R^{2}}\left(\beta_{m}^{2}+\frac{(2 n+1)^{2} \pi^{2}}{4 A^{2}}\right)\right]\right\}
\end{aligned}
$$

Note that Eq. (2c) consists of three parts: a spatially constant, time-dependent temperature ramp $(\mathrm{Ht})$, a spatially varying, time-invariant profile (function of cosine and Bessel functions), and a series of asymptotically time-decaying functions. After sufficiently long time, initial transients will have diminished and only the first and second terms will remain, resulting in a spatial profile which rises uniformly in time (i.e. all points in tandem) at a rate, $\mathrm{H}$. In this regime, the time invariant temperature profile at the cylinder mid-plane $(z=0$ location, where the cosine terms $\rightarrow 1)$ is given by subtracting off the boundary temperature, in other words: $\Delta T(r, 0)=T(r, 0, t)-T(R, 0, t)=T(r, 0, t)-H t$, as in Eq. (3):

$$
\Delta T(r, 0)=\frac{32 R^{2} A^{2}}{\pi}\left(\frac{Q_{0}}{\lambda}-\frac{H}{\alpha}\right) \sum_{n=0}^{\infty} \sum_{m=1}^{\infty} \frac{(-1)^{n}}{(2 n+1) \beta_{m}\left(4 A^{2} \beta_{m}^{2}+(2 n+1)^{2} \pi^{2}\right)} \frac{J_{0}\left(r \beta_{m} / R\right)}{J_{1}\left(\beta_{m}\right)}
$$

Note that in the limit as $A \rightarrow \infty$, the infinite cylinder solution (not a function of $z$ ) can be recovered. For instance, the temperature ramp solution $\left[Q_{0}=0\right]$ produces its expected quadratic profile as shown in Eq. (4), where we have made use of the convergent sums given by (5a) and (5b).

$$
\Delta T_{\text {ramp }, A-\infty}(r)=-\frac{8 H R^{2}}{\alpha \pi} \sum_{n=0}^{\infty} \sum_{m=1}^{\infty} \frac{(-1)^{n}}{(2 n+1) \beta_{m}^{3}} \frac{J_{0}\left(r \beta_{m} / R\right)}{J_{1}\left(\beta_{m}\right)}=-\frac{2 H R^{2}}{\alpha} \sum_{m=1}^{\infty} \frac{1}{\beta_{m}^{3}} \frac{J_{0}\left(r \beta_{m} / R\right)}{J_{1}\left(\beta_{m}\right)}=-\frac{H}{4 \alpha}\left(R^{2}-r^{2}\right)
$$




$$
\begin{gathered}
\sum_{n=0}^{\infty} \frac{(-1)^{n}}{(2 n+1)}=\frac{\pi}{4} \\
\sum_{m=1}^{\infty} \frac{1}{\beta_{m}^{3}} \frac{J_{0}\left(r \beta_{m} / R\right)}{J_{1}\left(\beta_{m}\right)}=\frac{1}{8}\left(1-\frac{r^{2}}{R^{2}}\right)
\end{gathered}
$$

The time-invariant temperature profile at the cylinder midplane $[z=0]$, Eq. (3) can be used to determine thermal diffusivity, $\alpha$, so long as the volumetric source term, $Q_{0}$, is negligibly small. In SITI experiments, temperatures along the mid-plane $[T(r, 0)]$ and boundary are measured. We also know the geometric quantities $\mathrm{R}$ and $\mathrm{A}$, and for temperature-ramped experiments, the heating rate $\mathrm{H}$ is also known. Furthermore, as long as temperatures are low enough, it is reasonable to assume that chemical heat releasing reactions are very slow, so $Q_{0}$ is approximately zero. Although the infinite series form of their solution presents certain challenges, Bessel functions are also known quantities. The only remaining unknown in Eq. (3) is the thermal diffusivity. Our approach will be to use Eq. (3) (or rather an accurate approximation thereof) to solve for the thermal diffusivity of energetic materials (EMs), of various types (i.e. explosives, pyrotechnics, or propellants) from temperature-ramped SITI data. Since we often have a good knowledge of material density and specific heat capacity from other sources (simple measurements, JANAF tables, etc), we can then obtain thermal conductivity from:

$$
\lambda=\alpha \rho C_{p}
$$

Our final task is to determine volumetric heat release in the EM contained within SITI at times when it is not negligibly small. In order to do this, we again appeal to Eq (3), this time requiring that $\lambda, H$, and $\alpha$ be known so that we can solve for $Q_{0}$.

\section{APPLICATION TO SITI GEOMETRY AND DATA}

The SITI devices have the capability of yielding spatially varying temperature profiles across the mid plane of the device, typically via nine TCs. Hence $T(r, 0, t)$ is known and by subtracting off the value of the ninth TC (closest to the boundary), one can obtain $\Delta T(r)$ at the radius $r$ of all the other TCs (later we will show that it is not necessary to assume that $\mathrm{TC}_{9}$ temperature $=$ boundary temperature). In the previous section, we derived the equation of the spatial profile at the mid plane, Eq. (3). However, since Eq. (3) is in the form of an infinite series, it will prove difficult to do this directly. Fortunately, we can approximate the form of Eq. (3) to a high level of accuracy with a much simpler form-a quadratic as given by Eq. (7a) [for $Q_{0}=0$ ], where $S$ is a dimensionless "scale factor." The analogy holds for the full $\left[Q_{0} \neq 0\right]$ form of Eq. (3) (combined ramp and volumetric source term) which yields Eq. (7b).

$$
\begin{gathered}
\text { Ramp Only } \\
\text { (inert material) }
\end{gathered}
$$

Ramp + Volumetric Heating (actively reacting material)

$$
\begin{gathered}
\Delta T=\frac{H S}{\alpha}\left(r^{2}-R^{2}\right) \\
\Delta T=\left(\frac{H}{\alpha}-\frac{Q_{0}}{\lambda}\right) S\left(r^{2}-R^{2}\right)
\end{gathered}
$$

Figure 4 shows dimensionless temperature profiles $\left(\Delta T \alpha / H R^{2}\right)$ for several different cylinder aspect ratios for both the full solution [Eq. (3) with $Q_{0}=0$, using the first 10,201 terms $(n=0$ to $100, m=0$ to 100 ) of the series] and the approximate quadratic solution, Eq. (7a). Listed in Fig. 4 are the $S$ values which give a best fit of Eq. (7a) to Eq. (3) $\left[Q_{0}=0\right]$ together with the r-squared statistic for goodness-of-fit. Note that for the infinite aspect ratio, the scaling factor is exactly 0.25 (with r-squared value of 1.0), consistent with the exact quadratic solution in Eq. (4). The goodness of fit for an aspect ratio of 1.0 corresponding to SITI is 0.9993 , indicating that a quadratic fit is still quite good (the local error is $<2 \%$ of the total $\Delta T \alpha / \mathrm{HR}^{2}$ at any point in the profile). The scaling factor, $\mathrm{S}$, for an aspect ratio of 1.0 is 0.203 . 
At this point, it should be mentioned that the finite cylinder formulas shown here assume that the boundary condition (e.g. temperature ramp) is applied over the entire surface of the EM, including the ends. For the HOT-SITI device shown in the right side of Fig. 1 , this is a good assumption since the high conductivity aluminum confinement material contacts the EM all the way around. For the original SITI device (left side of Fig. 1), the assumption is less valid since a part of the upper and lower surfaces of the EM are exposed to an expansion gap. This gap was included in the SITI design to allow for thermal expansion of the EM, while allowing a hermetic seal to be maintained in the device. The gap was not required in HOT-SITI because the pyrotechnic materials to be tested all had a significant amount of internal void space, and thermal expansion can be accomodated by "crushing out" internal voids.

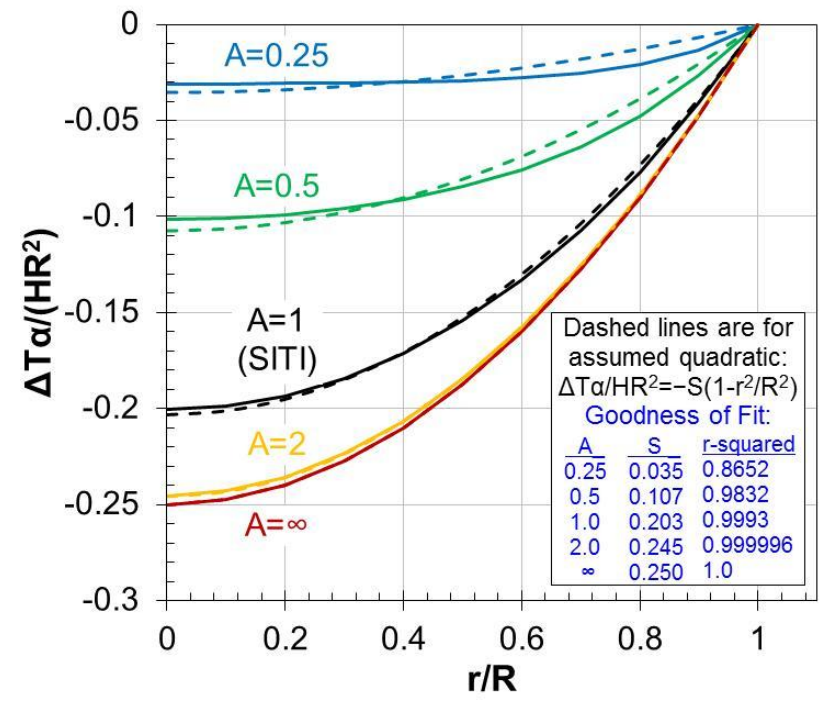

Fig. 4. Temperature Profiles for Various Cylinder Aspect Ratios

To determine the effect of the gap, simple simulations were run using a finite element code in which the boundary temperature was ramped over a geometry with an aspect ratio of 1.0. The mid-plane temperature profile was sampled and fit to the form of Eq. (7a). For a case with no gap (HOT-SITI), the expected $S=0.203$ scaling factor was recovered. For a case with a gap filled with a perfect insulator the $S$ factor of 0.246 was obtained with a $0.999999 \mathrm{r}$-squared value, indicating that the profile is represented very well by a quadratic. For cases in which the gap was filled with either air (i.e. a solid pellet with air above and below it, representing conduction heat transfer plus radiation across the gap) or EM (i.e. a powdered material fills the entire cavity) the $S$ factor depends on the gap height (tests have been conducted with six different gap heights). Table II shows the S factors for the various versions of SITI. In the table figures, yellow is the EM, red lines indicate the location of the applied temperature ramped BC and the dotted lines are the TC locations at the midplane. Note that for most of the tests with an air gap, the $S$ factor is very close to 0.243 ; it is slightly lower when the gap was filled with an EM. Most SITI tests were conducted with gaps of 0.125 " $(3.18 \mathrm{~mm})$ or 0.150 " $(3.81 \mathrm{~mm})$ so the expected S factor would be $\sim 0.226$ for EM pellets with air space and $\sim 0.223$ for powders which fill the cavity volume. The observation that the $S$ factor for air-filled gaps are smaller than EM-filled gaps for large gap sizes (implying that the air-cases permit more heat transfer, being farther away from the $S$ factor for perfect insulation cases) is attributed to the inclusion of radiation heat transfer across the gap in the air-filled cases.

Table II: S Factors for Various Versions of SITI

\begin{tabular}{|c|c|c|c|c|c|c|c|}
\hline HOT-SITI: & S factor & SITI: & $\begin{array}{c}\text { gap } \\
\text { height } \\
\text { inch [mm] }\end{array}$ & $\begin{array}{c}\% \text { free } \\
\text { volume }\end{array}$ & $\begin{array}{c}\text { S factor } \\
\text { (air } \\
\text { in gap) }\end{array}$ & $\begin{array}{c}\text { S factor } \\
\text { (EM } \\
\text { in gap) }\end{array}$ & \begin{tabular}{|c} 
S factor \\
(insulated \\
gap)
\end{tabular} \\
\hline \begin{tabular}{l}
$1.0 "$ \\
$(2.54 \mathrm{~cm})$ \\
\hdashline$\ldots \ldots \ldots \ldots \ldots .$. \\
$0.5 "$ \\
$(1.27 \mathrm{~cm})$
\end{tabular} & 0.2033 & 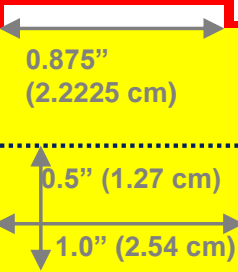 & $\begin{array}{l}0.063[1.60] \\
0.125[3.18] \\
0.150[3.81] \\
0.218[5.54] \\
0.650[16.5] \\
1.959[49.8]\end{array}$ & $\begin{array}{c}8.8 \% \\
16.1 \% \\
18.7 \% \\
25.0 \% \\
50.0 \% \\
75.0 \%\end{array}$ & $\begin{array}{l}0.2195 \\
0.2255 \\
0.2268 \\
0.2289 \\
0.2307 \\
0.2307\end{array}$ & $\begin{array}{l}0.2147 \\
0.2224 \\
0.2247 \\
0.2297 \\
0.2390 \\
0.2399\end{array}$ & $\begin{array}{l}0.2463 \\
0.2463 \\
0.2463 \\
0.2463 \\
0.2463 \\
0.2463\end{array}$ \\
\hline
\end{tabular}


The equations developed above state that after the initial transient has ended, the established relative temperature profile can be approximated very well by a quadratic function that remains constant. In other words, it simply rises in tandem with every point moving upward at the same rate as the boundary. Equation (7a) can be used to obtain the thermal diffusivity, $\alpha$. This is done by fitting data to a quadratic form such as $\Delta T=C_{1} r^{2}+C_{2}$ and then associating the coefficient $C_{1}$ with the appropriate term in Eq. (7a) (e.g. $\mathrm{C}_{1}=\mathrm{HS} / \alpha$ ), at which point it is a simple matter to obtain Eq. (8a). Thus if spatially varying temperatures are available from a ramp-heated experiment, $\alpha$ can be determined directly from a simple curve fit of the data and Eq. (8a). A similar approach can be used with the superposed (ramp and volumetric source term) case to obtain Eq. (8b) which can be used to solve for the volumetric source term. This requires that thermal conductivity be known.

$$
\begin{array}{cc}
\begin{array}{c}
\text { Ramp Only } \\
\text { (inert material) } \\
\begin{array}{c}
\text { Ramp + Volumetric Heating } \\
\text { (actively reacting material) }
\end{array}
\end{array} & \alpha=\frac{H S}{C_{1}} \\
Q_{0} & =\frac{H \lambda}{\alpha}-\frac{C_{1} \lambda}{S}=H \rho C_{p}-\frac{C_{1} \lambda}{S}
\end{array}
$$

\section{APPLICATION TO HOT-SITI FOR PYROTECHNIC MATERIALS}

Data from HOT-SITI tests of two different materials (TKP output and $\mathrm{KClO}_{4}$ ) were obtained and processed to obtain thermal diffusivity and thermal conductivity (for both inert and reactive materials), and heat release (for reactive materials). The data reduction is accomplished with a simple four-step process.

\section{STEP 1. BIAS CORRECTION.}

First the TC data are corrected for bias. This is done by collecting temperature data prior to the device heater being turned on (when all TCs should be reading the same room-temperature value) over a short period of time. Subtracting each individual TC's time-averaged value from the overall group time-averaged value yields the TC bias. These bias values were typically on the order of $\pm 0.15^{\circ} \mathrm{C}$ (see Fig. 5). The bias values were than subtracted from the temperatures of each of the nine interior TCs. The bias values for the various TC locations shown in Fig. 5 for two separate runs (Run 642 with TKP output material and Run 645 with $\mathrm{KClO}_{4}$ ) are remarkably similar to each other. This would be an unexpected result, if the bias is truly a random variable. However, from test to test the TC of the same location (such as $\mathrm{TC}_{1}$ at the center) is

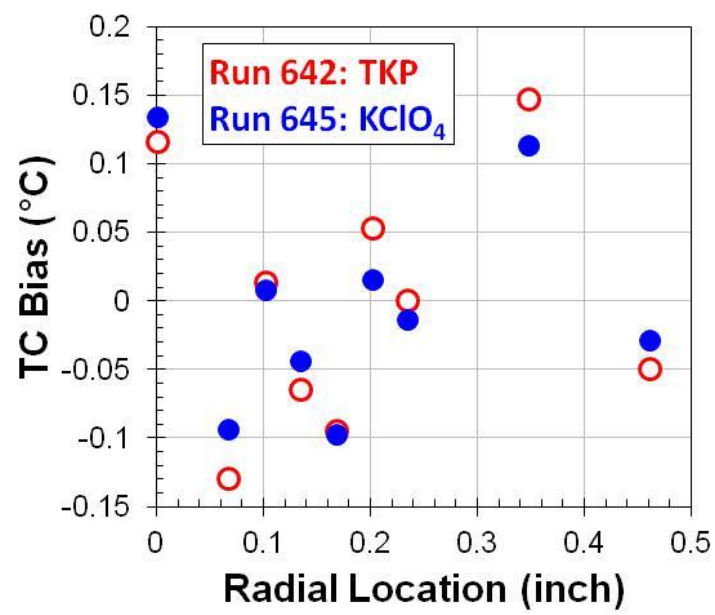

Fig. 5: TC bias for two HOT-SITI tests. connected to the same extension wire and same location in the signal processing circuitry (i.e. $\mathrm{TC}_{1}$ is always connected in the same way to the same parts for every test). Hence the bias present in the TC readings in Fig. 5 seems to be relatively reproducible. Furthermore, the temperature precision (location to location variation) is quite good. This will be important for the analysis that will be performed here.

\section{STEP 2. THERMAL DIFFUSIVITY DETERMINATION FROM QUADRATIC TEMPERATURE FIT}

After correcting the TCs for bias, the second step is to fit the data to a quadratic form of $T=C_{1} r^{2}+$ $\mathrm{C}_{2}$. This is done in a least-squares approach, i.e. we are minimizing the objective function $f$ in Eq. (9) which can be done quite easily using commonly available regression techniques. The least squares fit to yield $\mathrm{C}_{1}$ and $\mathrm{C}_{2}$ are given in (10). 


$$
\begin{aligned}
& f=\sum_{i=1}^{n}\left[T_{i}-\left(C_{1} r_{i}^{2}+C_{2}\right)\right]^{2} \\
& \mathrm{C}_{1}=\frac{\mathrm{n} \sum_{\mathrm{i}=1}^{\mathrm{n}} \mathrm{r}_{\mathrm{i}}^{2} \mathrm{~T}_{\mathrm{i}}-\sum_{\mathrm{i}=1}^{\mathrm{n}} \mathrm{r}_{\mathrm{i}}^{2} \sum_{\mathrm{i}=1}^{\mathrm{n}} \mathrm{T}_{\mathrm{i}}}{\mathrm{n} \sum_{\mathrm{i}=1}^{\mathrm{n}} \mathrm{r}_{\mathrm{i}}^{4}-\sum_{\mathrm{i}=1}^{\mathrm{n}} \mathrm{r}_{\mathrm{i}}^{2} \sum_{\mathrm{i}=1}^{\mathrm{n}} \mathrm{r}_{\mathrm{i}}^{2}} \quad \mathrm{C}_{2}=\frac{\sum_{\mathrm{i}=1}^{\mathrm{n}} \mathrm{T}_{\mathrm{i}}-\mathrm{C}_{1} \sum_{\mathrm{i}=1}^{\mathrm{n}} \mathrm{r}_{\mathrm{i}}^{2}}{\mathrm{n}}
\end{aligned}
$$

Note that we are fitting the temperatures $\left(T_{i}\right)$ directly instead of the temperature differences relative to the boundary at $r=R$ (i.e. $\Delta T_{i}=T_{i}-T_{R}$ ). This is because $T_{R}$ is not known a priori (there is a small unknown temperature drop between the aluminum wall and the EM due to contact resistance) and assuming that the temperature $T_{9}$ from the outermost (ninth) $T C$, is exactly equal to $T_{R}$ over-weights its effect (i.e. $T_{9}$ would then appear in every $\Delta T$ term such as $\Delta T_{1}=T_{1}-T_{9}, \Delta T_{2}=T_{2}-T_{9}$, etc.). Solving in the form of Eq. (9) allows us to include the possibility that $T_{9}$ is not exactly equal to $T_{R}$ and weights each TC equally. Once the coefficients $\mathrm{C}_{1}$ and $\mathrm{C}_{2}$ are solved, $\mathrm{T}_{\mathrm{R}}$ can be obtained. Only the constant term $\mathrm{C}_{2}$ is affected by whether one fits $\Delta T$ or $T$; the quadratic coefficient $C_{1}$ is the same in either regard. Once $C_{1}$ is obtained, one can directly obtain thermal diffusivity, $\alpha$, from Eq. (8a), since the ramp rate, $\mathrm{H}$, and the scaling factor, S, are known.

Figure 6 shows temperature data and associated quadratic curve fits from two HOT-SITI tests. The left column shows results from a test with TKP output powder (Run 642). The right column figures are for $\mathrm{KClO}_{4}$ (Run 645). Individual graphs show results at various times. All of the vertical scales are the same, spanning a range of $3^{\circ} \mathrm{C}$ except the last graph for TKP at $442.9 \mathrm{~min}$. At that time, internal heat generation results in an inversion of the temperature profile. Note that the quadratic curve fits do a respectable job of fitting the data-even the inverted profile. 

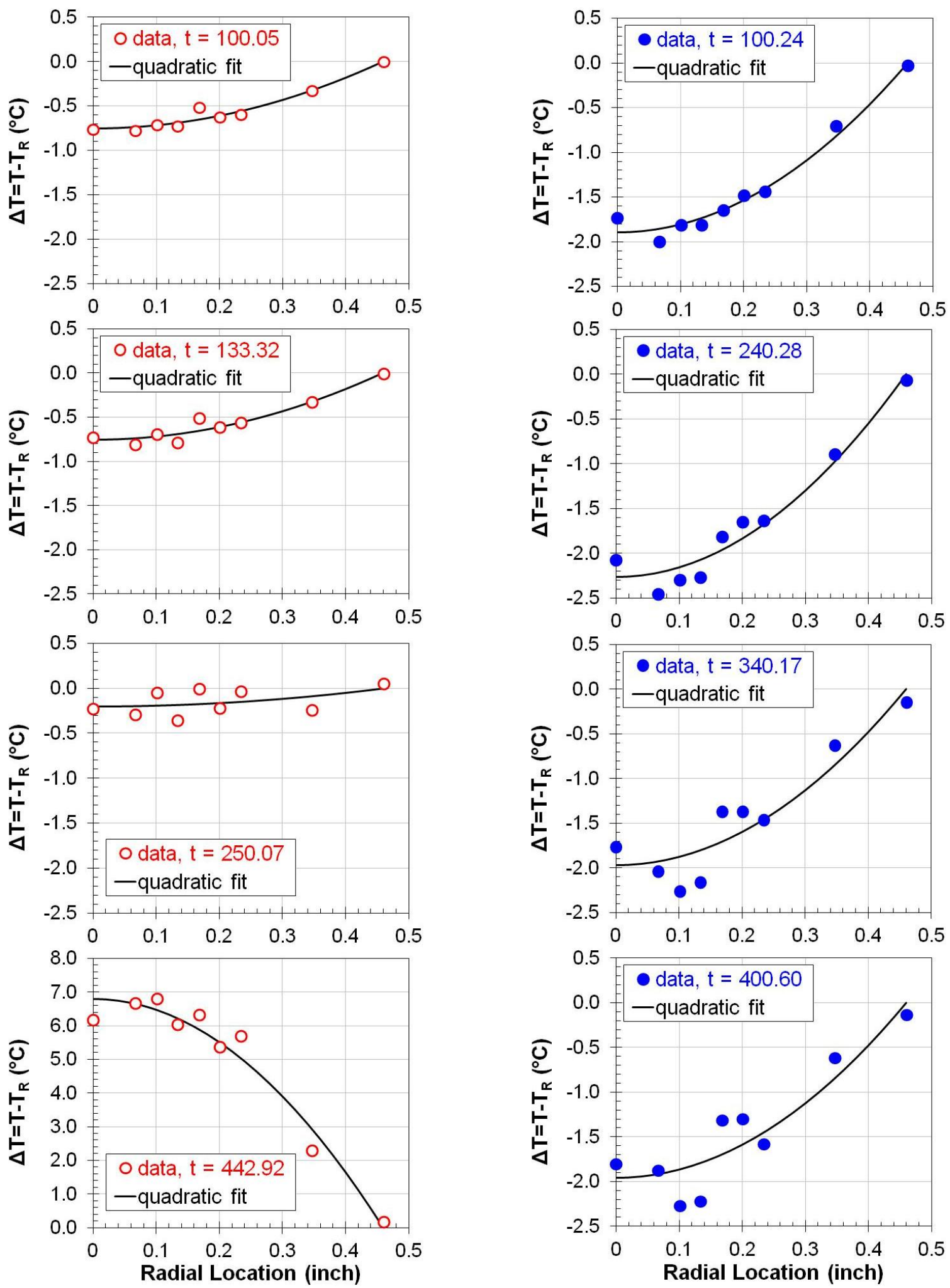

Fig. 6: Thermocouple data from two different HOT-SITI experiments and quadratic curve fits. Left column is for TKP output powder (Run 642); right column is for $\mathrm{KClO}_{4}$ (Run 645). The heating rate was $1^{\circ} \mathrm{C} / \mathrm{min}$ for both tests. Labels indicate the time (in minutes) corresponding to the data set. 


\section{STEP 3. THERMAL CONDUCTIVITY DETERMINATION}

The third step is to obtain thermal conductivity. Once thermal diffusivity has been determined, conductivity can be obtained by multiplying by density and heat capacity $\left(\lambda=\alpha \rho C_{p}\right)$. For the two materials tested here the density was measured $\left(2477 \mathrm{~kg} / \mathrm{m}^{3}\right.$ for TKP and $2092 \mathrm{~kg} / \mathrm{m}^{3}$ for $\mathrm{KClO}_{4}$ ) from the pellet mass and size. (Note that both of these were porous material densities. The full material densities are 3079 and $2524 \mathrm{~kg} / \mathrm{m}^{3}$ for TKP and $\mathrm{KClO}_{4}$ respectively). The specific heat capacities of $\mathrm{Ti}$ and $\mathrm{KClO}_{4}$ are known from literature [10]. For TKP, a mass weighted average $(41 \% \mathrm{Ti}$, $59 \% \mathrm{KClO}_{4}$ ) of the constituents' specific heats was computed. Figure 7 shows the heat capacities of the two materials. The abrupt change in specific heat for the TKP output material is due to a phase change in $\mathrm{Ti}$ at $893^{\circ} \mathrm{C}$.

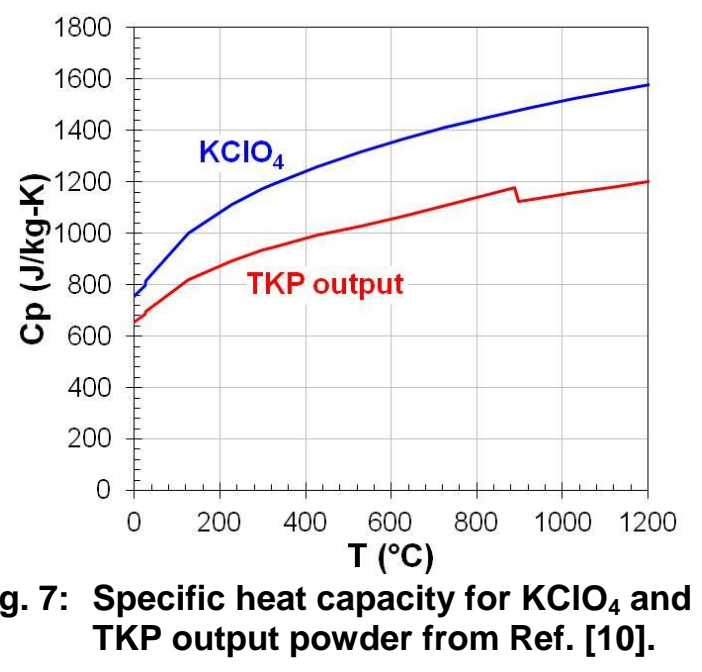

Two data sets, Run 642 (TKP output) and Run $645\left(\mathrm{KClO}_{4}\right)$ were considered for analysis. Figures 8 and 9 represent the interior temperatures from those two runs. In each of these, the left graphs show the base temperatures and the right graphs show the temperatures relative to $\mathrm{TC}_{9}$ (outermost interior TC).

The temperatures shown in these figures are nearly perfectly overlaid during much of the heating profile (left graphs). By subtracting off the ramp (accomplished by plotting data relative to $\mathrm{TC}_{9}$ as in the right graphs of Figs. 8 and 9) the variations become apparent. Of note is the orthorhombic-to-cubic phase change of $\mathrm{KClO}_{4}$ occurring near times of $300 \mathrm{~min}$ in both figures. Also of interest is the change in slope in Fig. 8 beginning at about 150 min, which progresses and ultimately results in interior temperatures higher than boundary temperatures and a thermal runaway. We surmise that chemical reactions are beginning to occur at about 150 min into Run 642 (TKP). In contrast, Fig. 9 appears to show no similar reaction activity for the Run $645 \mathrm{KClO}_{4}$ test. The jumps present in $\mathrm{TC}$ traces beyond about $420 \mathrm{~min}$. in Fig. 9 are believed to be associated with the TCs shifting and/or shorting.

The temperature data from Runs 642 and 645 were analyzed using the process steps 1 to 3 described above. The results are shown in Figs. 10 and 11 which give thermal diffusivity and thermal conductivity as a function of time and temperature, respectively. In these graphs, the red (TKP) and blue 

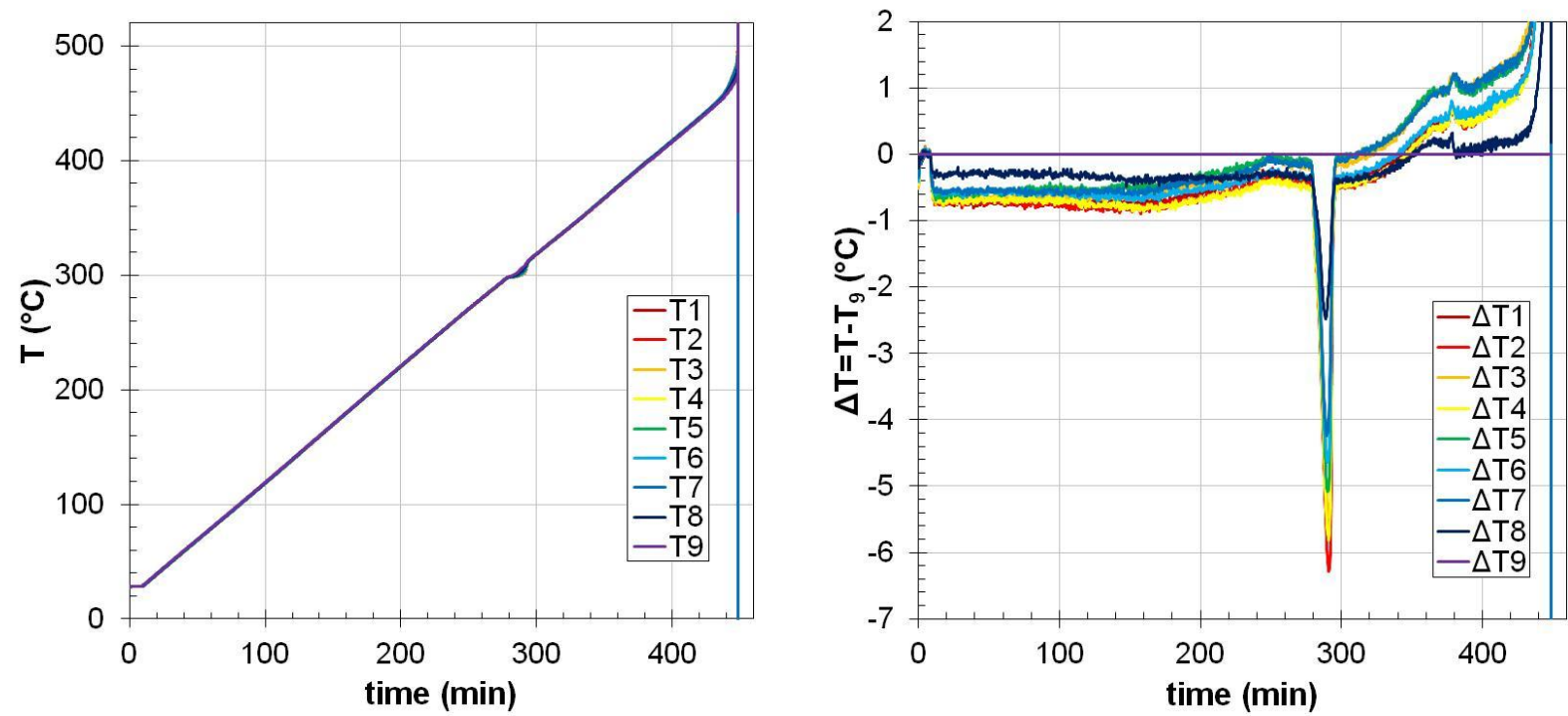

Fig. 8: HOT-SITI Run 642 (TKP output) interior temperatures. Left: T, Right: T relative to TC .
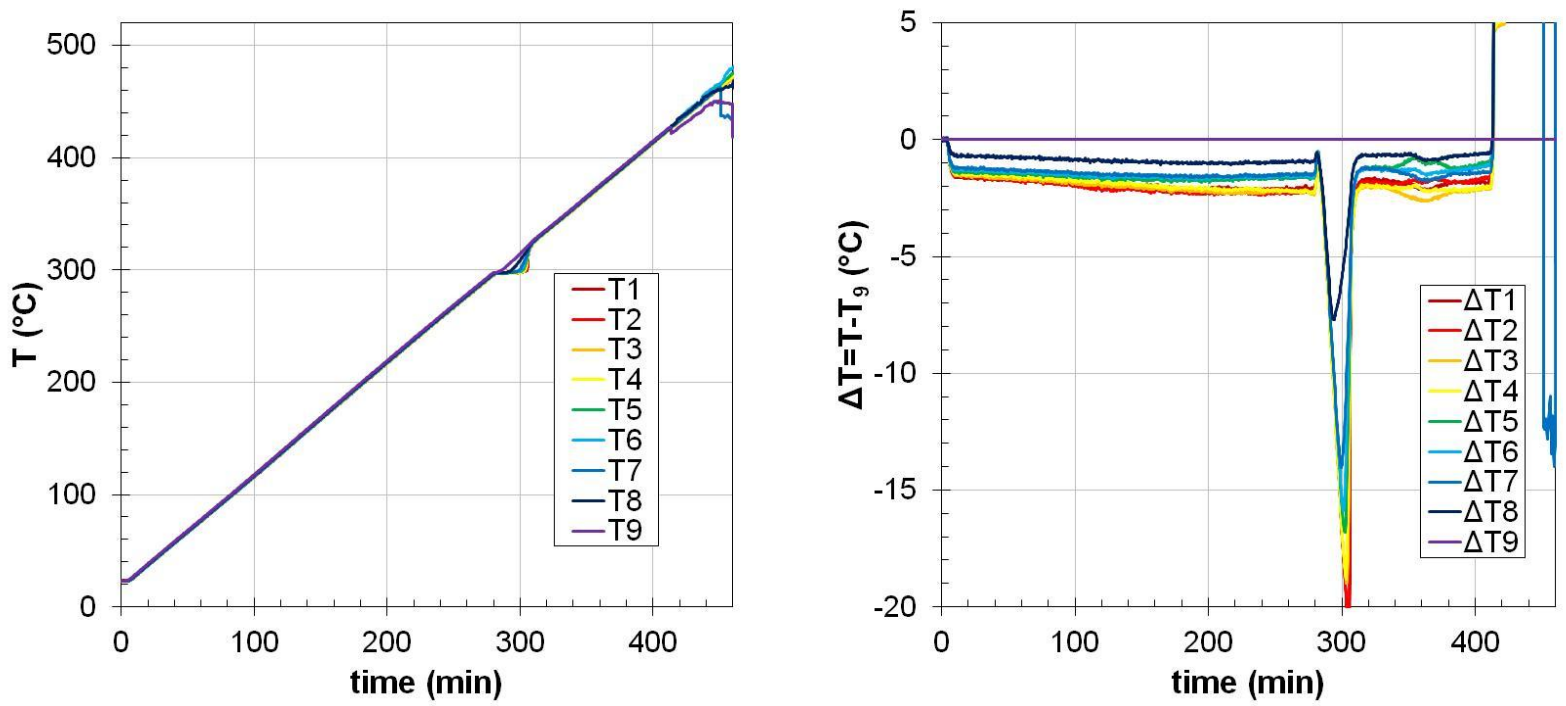

Fig. 9: HOT-SITI Run $645\left(\mathrm{KCIO}_{4}\right)$ interior temperatures. Left: $\mathrm{T}$, Right: T relative to $\mathrm{TC}_{9}$.

$\left(\mathrm{KClO}_{4}\right)$ represent results in which the approach is believed to be valid, while gray represents results which are not valid because of thermal events such as phase changes or chemical reactions which lead to changes in the shape of the temperature profiles, so that Eq. (8a) is no longer appropriate. During the reactive portion of the tests, Eq. (8b) can be used as described later.

The results of Figs. 10 and 11 indicate nearly constant or moderately changing values of thermal diffusivity and conductivity. The spread in the data is due to slight changes in temperatures at the TCs from step to step which result in changes in the quadratic curve fit. The spread for the TKP output material appears larger than for $\mathrm{KClO}_{4}$. This is probably due to the fact that the overall temperature profile is generally smaller for TKP than for $\mathrm{KClO}_{4}$ (compare the $\mathrm{t}=100$ min data from Fig. 6 , for instance), so TC "noise" will be magnified-it is a larger fraction of the overall signal (here the overall "signal" is the height of the quadratic profile). This also suggests that since heating at a faster rate (say 3 or $10^{\circ} \mathrm{C} / \mathrm{min}$ ) will produce a greater profile height (Eq. (7a) shows the height of the profile is directly proportional to the heating rate $\mathrm{H}$ ), the signal to noise ratio might be improved by testing at a somewhat faster rate, with the potential drawback that the initial transient (the decaying exponential terms of Eqs. (2)) would invalidate the approach during a fractionally larger portion of the available data. Incidentally, the 1/e time constant 
for the truncated cylinder, $\tau=4 \mathrm{R}^{2} \mathrm{~A}^{2} /\left(\alpha\left(4 \mathrm{~A}^{2} \beta_{\mathrm{m}}{ }^{2}+(2 n+1)^{2} \pi^{2}\right)\right)$ from the forms in Eqs. (2) were evaluated for the longest $(\mathrm{m}=1, \mathrm{n}=0)$ mode with the diffusivity values obtained here $\alpha=2 \times 10^{-7} \mathrm{~m}^{2} / \mathrm{s}$ for $\mathrm{KCIO}_{4}$ and $6 \times 10^{-7}$ $\mathrm{m}^{2} / \mathrm{s}$ for TKP output. These yielded time constants on the order of $98 \mathrm{~s}$ for $\mathrm{KClO}_{4}$ and $33 \mathrm{~s}$ for TKP output. After 3 time constants, $95 \%$ of the transient has damped out so we expect the quadratic profile assumption to be valid by $\sim 5 \mathrm{~min}$. for $\mathrm{KClO}_{4}$ and $\sim 1.5 \mathrm{~min}$. for TKP, if not sooner. This is only a very small portion of the test at $1^{\circ} \mathrm{C} / \mathrm{min}$.

The traces in Figs. 10 and 11 for $\mathrm{KClO}_{4}$ indicate two phases, orthorhombic at low temperature and cubic at higher temperature. There is a $15 \%$ reduction in crystal density associated with the phase change [11], so the cubic phase occupies $15 \%$ greater volume than the orthorhombic. Here the material started out at $\sim 83 \%$ of the orthorhombic theoretical maximum density, and with the phase change it converts to $\sim 98 \%$ of the cubic theoretical maximum density, implying that pore volume has been crushed out. We also note that the thermal expansion of $\mathrm{KClO}_{4}$ is fairly high, $130 \mu \varepsilon / \mathrm{K}$ [12], compared to the 23 $\mu \varepsilon / \mathrm{K}$ for aluminum. So there should be several percent additional volume change which will probably fill the entire available space. Material lockup and deformation may have resulted in the strange TC behavior beyond 420 min seen in Fig. 9.
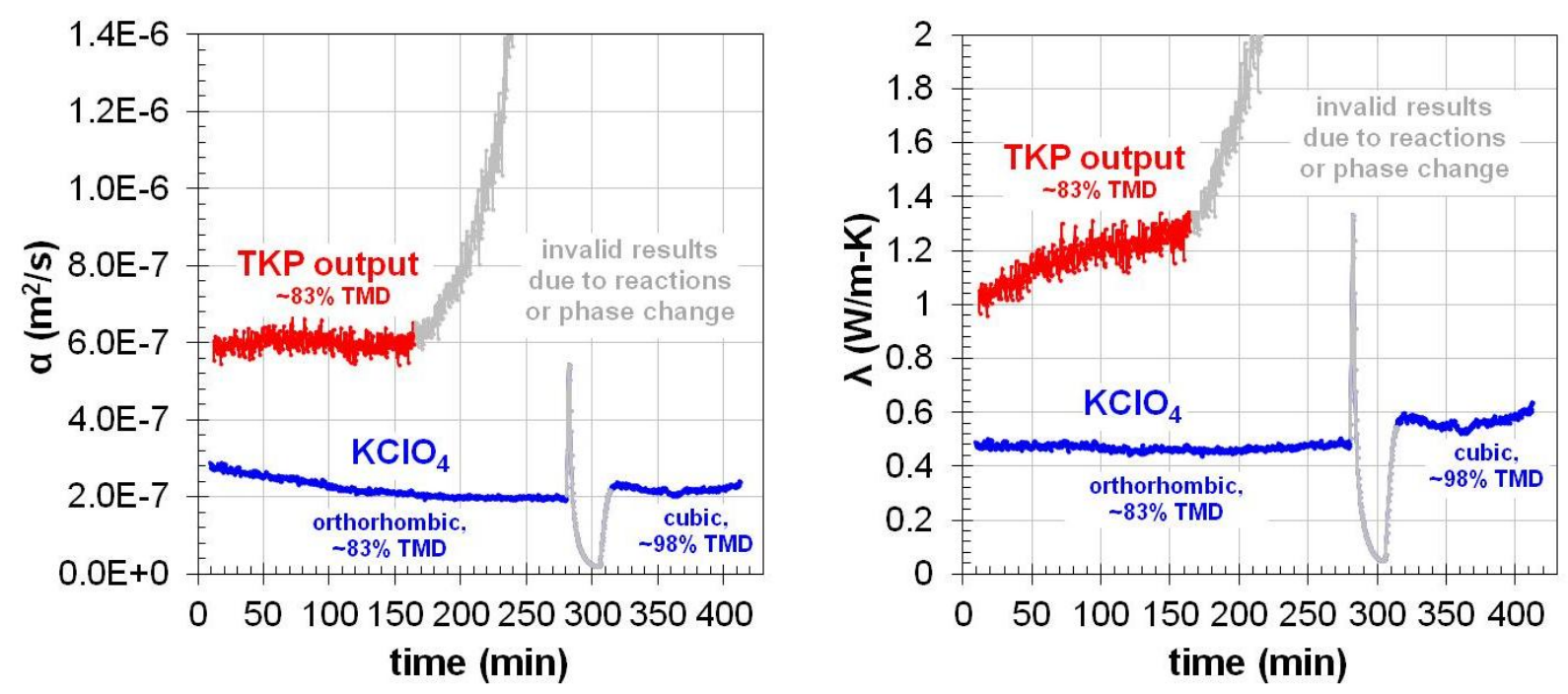

Fig. 10:Thermal diffusivity (left) and thermal conductivity (right) as a function of time obtained from processing data from HOT-SITI Runs 642 (TKP output) in red and $645\left(\mathrm{KClO}_{4}\right)$ in blue. Grayed out data indicate thermal events (reactions and/or phase changes) which invalidate the approach. 

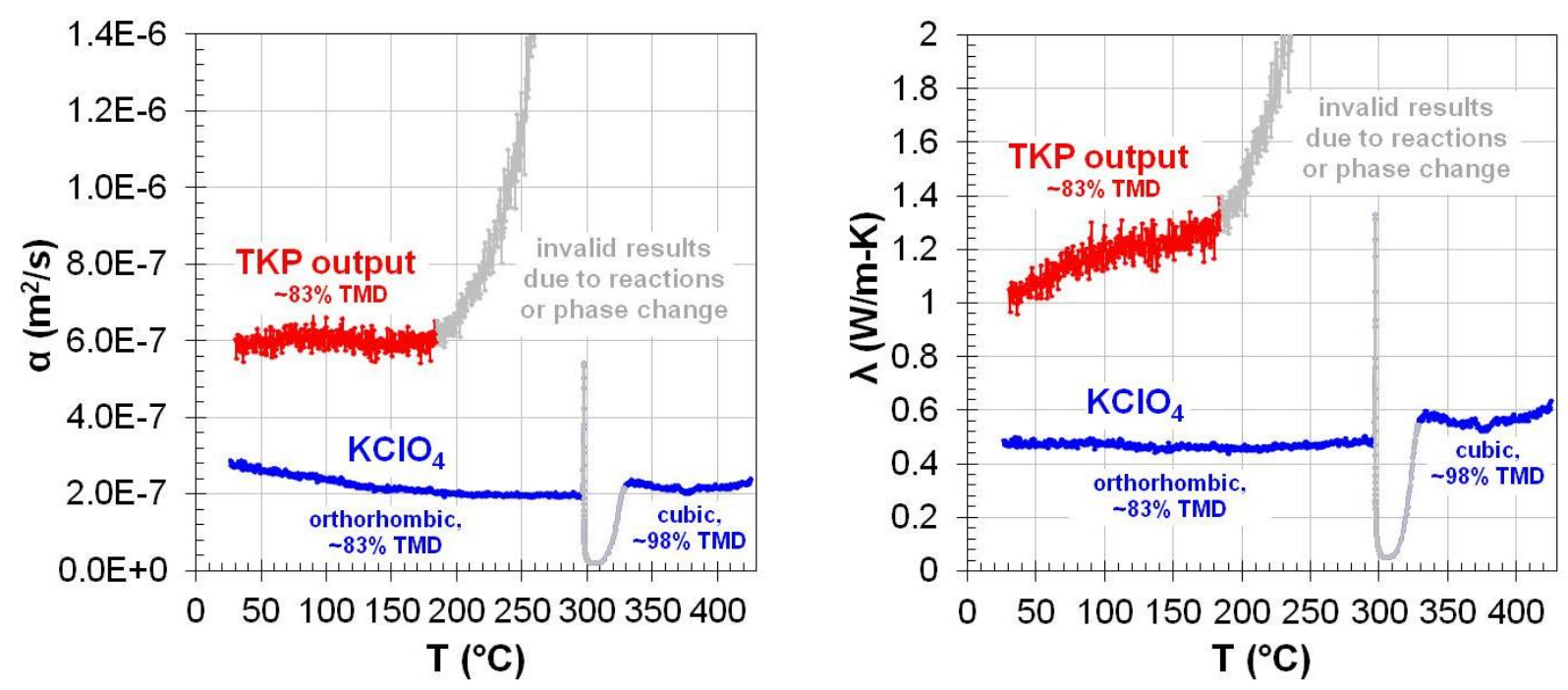

Fig. 11: Thermal diffusivity (left) and thermal conductivity (right) as a function of temperature obtained from processing data from HOT-SITI Runs 642 (TKP output) in red and 645 $\left(\mathrm{KClO}_{4}\right)$ in blue. Gray data indicate thermal events (reactions and/or phase changes) which invalidate the approach.

There are a few available literature values for the thermal conductivity of $\mathrm{KClO}_{4}$. Figure 12 shows a comparison of thermal conductivity values determined here along with results of two other references [13], [14] as a function of material density. The current work gave a range of data for orthorhombic $\mathrm{KClO}_{4}$ at $\sim 83 \% \mathrm{TMD}$ of 0.44 to $0.51 \mathrm{~W} / \mathrm{m}-\mathrm{K}$ with an average of $0.47 \mathrm{~W} / \mathrm{m}-\mathrm{K}$. For cubic $\mathrm{KClO}_{4}$ at $\sim 98 \% \mathrm{TMD}$, the range was 0.52 to $0.64 \mathrm{~W} / \mathrm{m}-\mathrm{K}$ with an average of $0.57 \mathrm{~W} / \mathrm{m}-\mathrm{K}$. The literature values were taken at lower density levels and the temperatures tested in the literature references were lower: Ref. [13] reported data from 0 to $50^{\circ} \mathrm{C}$; Ref. [14] tested up to $100^{\circ} \mathrm{C}$. It is noteworthy that a variety of techniques were used to determine conductivity. These included temperature modulated DSC [14], a hot wire approach [14] and a guarded heat flow device [13]. Error estimates were not provided in any of these references. While we cannot exactly make direct comparison, the trends projected in Fig. 12 show that our measurements reasonably compare with literature data. In that figure we have plotted the nominal value (symbols) and the range (bars) for each density.

Specific heat data as a function of temperature were available from literature (e.g. Fig. 7) allowing us to obtain thermal conductivity. If these data were not available, the diffusivity could still be obtained but conductivity could not. For materials such as organic explosives with polymeric binders, heat capacity as a function of temperature is often unavailable, and measurements (perhaps with differential scanning calorimetry or other technique) would be required.

\section{STEP 4. DETERMINATION OF HEAT RELEASE}

The fourth step of the process is to obtain the volumetric heat release. This requires additional

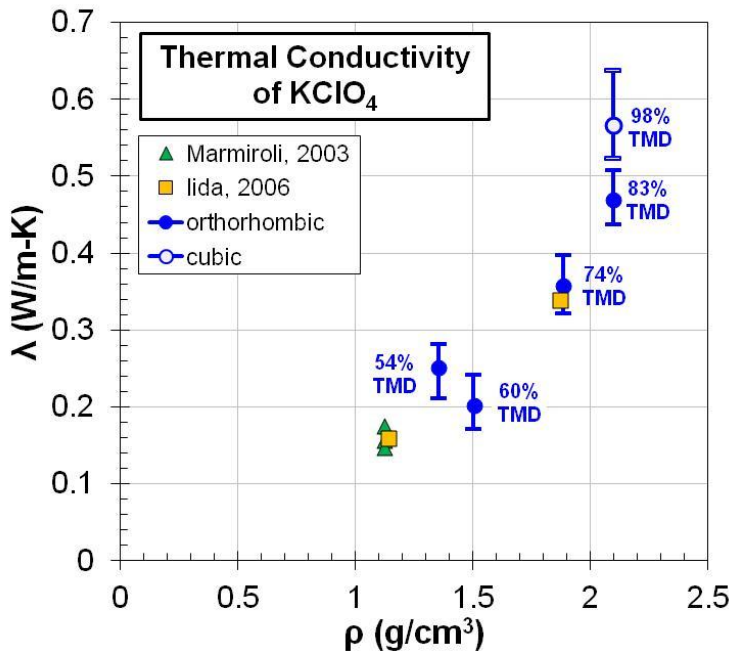

Fig. 12: Thermal conductivity of two phases of $\mathrm{KClO}_{4}$ determined in the current work and data from Refs. [13], [14]. information beyond the previous steps. For instance, we have seen that so long as there is no internal heat generation, thermal diffusivity can be obtained simply from quadratic curve fits and Eq. (8a). Thermal conductivity requires diffusivity along with density and specific heat and Eq. (6). Now to get 
volumetric heat release, one can use Eq. (8b) but this presupposes that one knows thermal conductivity already; we cannot solve for heat release and thermal diffusivity/conductivity at the same time since the two quantities are confounded. Our approach then is to use the diffusivity/conductivity data taken at inert conditions (when reactions are negligible) and extrapolate them to the higher temperature portions at the test when reactions have begun. In the case of TKP output powder, the diffusivity data is nearly constant at $\alpha=6 \times 10^{-7} \mathrm{~m}^{2} / \mathrm{s}$ until about $180^{\circ} \mathrm{C}$ at which point reactions begin to occur. If we assume that $\alpha$ remains constant at that same value beyond $180^{\circ} \mathrm{C}$, we can obtain the conductivity, $\lambda$ at high temperatures as well and thereby have sufficient information to use Eq. (8b). Another inherent assumption is that the heat release is indeed uniformly distributed over the volume. This may be approximately true so long as temperatures do not vary much throughout the domain (assuming temperature dependent reaction rates). But if temperatures become disparate, we expect that assumption to break down.

Figure 13 shows heat release computed from Eq. (8b) for HOT-SITI Run 642 with TKP output powder. Both instantaneous heat release rates and cumulative total heat release are plotted. The left side graph shows the raw heat release as a function of time. Notice that heat release begins to occur at about 150 min into the experiment and then turns negative at about $280 \mathrm{~min}\left(300^{\circ} \mathrm{C}\right)$. This downturn is associated with the endothermic $\mathrm{KClO}_{4}$ phase change $\left(\sim 99.4 \mathrm{~kJ} / \mathrm{kg}\right.$ of $\mathrm{KClO}_{4}$ or $\sim 58.6 \mathrm{~kJ} / \mathrm{kg}$ of TKP), since Eq. (8b) does not differentiate between an exothermic and endothermic process in computing heat release.

If the data are corrected to determine just the "reactive" portion of the energy release (phase change energy is added back in) the result is a monotonic cumulative energy release curve as shown on the right hand side of Fig. 13, in this case plotted with respect to average temperature instead of time. The "rollover" of the cumulative energy release curve which appears in the right graph (and does not appear in the left side graph) is probably associated with the fact that energy is being released very quickly leading to rapid temperature rises locally which cannot be dissipated by conduction to the boundary. Hence it doesn't take as much cumulative energy release to effect a similar temperature rise since the confinement material doesn't have to be heated.
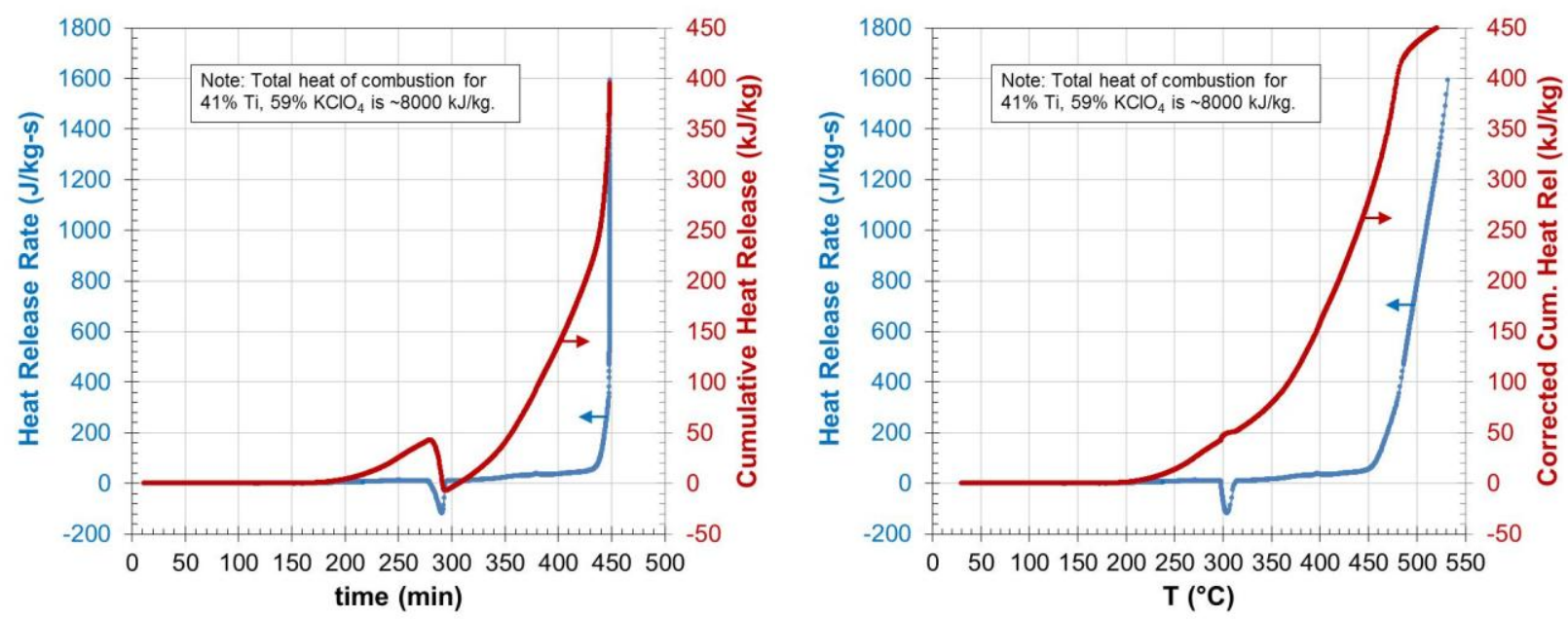

Fig. 13: Heat release as determined for HOT-SITI Run 642 (TKP output). Blue lines represent heat release rate $[\mathrm{J} / \mathrm{kg}-\mathrm{s}]$, red lines represent cumulative heat release $[\mathrm{kJ} / \mathrm{kg}]$. Left: raw heat release as a function of time. Right: corrected heat release showing just reactive portion (accounting for phase change energy) as a function of average material temperature. The data sampling rate was adjusted during this test with slower rates $(0.05 \mathrm{~Hz})$ used early in the test when reaction rates were slow and faster rates $(5 \mathrm{~Hz})$ later in the test when reactions were fast.

As a cross check or validation of the approach, a finite element simulation of HOT SITI Run 642 was performed. The external temperature boundary condition applied was obtained from the experimental data. Thermophysical properties of the pyrotechnic material were used as derived from the 
approach above. And a volumetric source term derived from this method (e.g. Fig. 13) was applied uniformly over the pyrotechnic volume. Interior temperatures corresponding to the thermocouple locations were then monitored over time. Figure 14 shows a comparison of the temperatures obtained from the simulation with those obtained from the experiment. The external edge temperature was subtracted off to eliminate the temperature ramp and highlight the spatial variations. Note that the heat release obtained here does a fairly respectable job of reproducing the experimental results, lending credence to this approach.
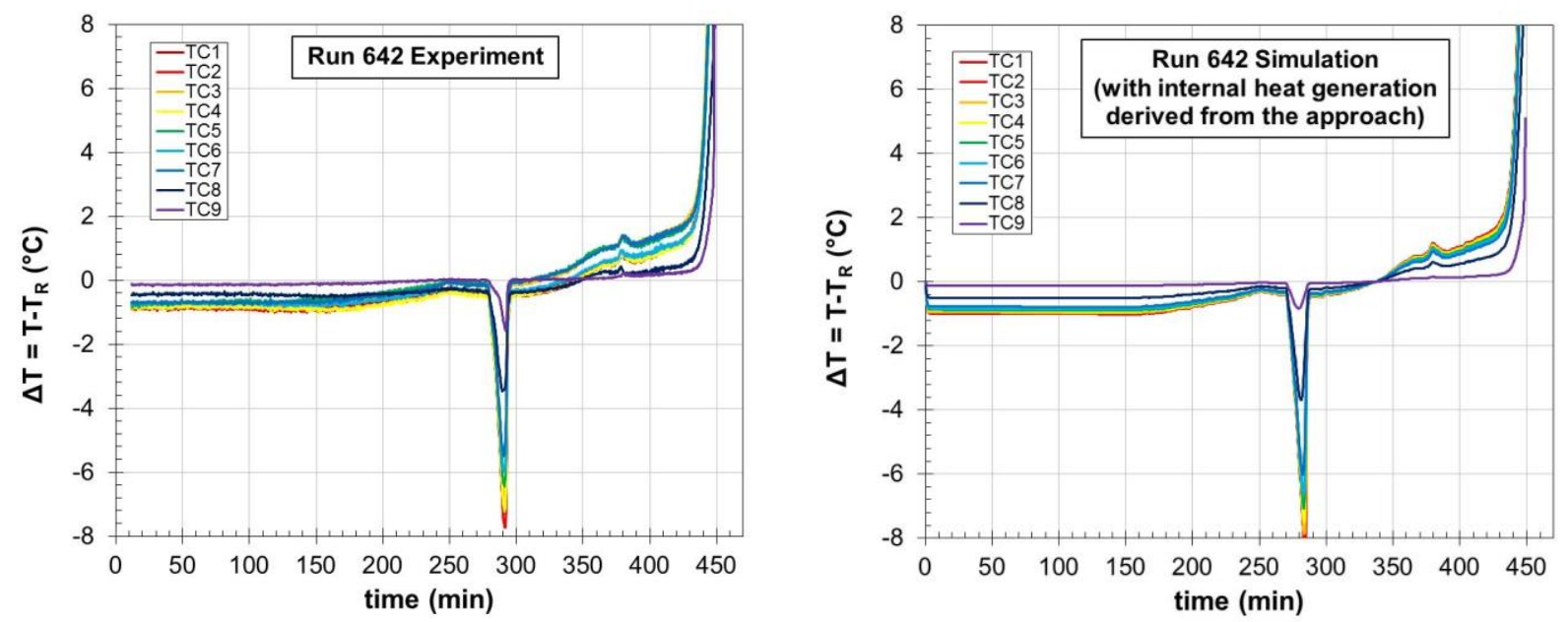

Fig. 14: Temperature differences (T-T $T_{R}$, where $T_{R}$ is at the outer edge of the material) from HOT-SITI Run 642. Left: Experimental temperature; Right: Simulated using a finite element model with heat release derived from this approach (Fig. 13) used as a volumetric source term.

\section{APPLICATION TO OTHER PROPELLANTS AND EXPLOSIVE MATERIALS}

The approach described above for pyrotechnic materials can also be followed for other energetic materials. It can also be applied to other heating profiles, though adjustments must be made. For instance, SITI tests are often conducted with a ramp-and-hold temperature profile in which the device is heated rapidly (typically $10 \mathrm{~min}$. total ramp time) from room temperature to a hold temperature. Since the ramp period is so short, the initial transients may not fully dampen out until the ramp is nearly over, thus reducing the amount of data available for analysis. However, during the hold period, Eq. (8b) can still be applied to analyze heat release, with the ramp rate term $(\mathrm{H})$ set to zero. Here we show some examples of the data analysis procedure applied to various energetic materials.

\section{COMPOSITE PROPELLANT A.}

Propellant A is an aluminized AP / HTPB composite solid propellant. Samples of this propellant of two different types were tested in SITI: original, full-density propellant and shredded propellant at $52 \%$ density. Ramp-and-hold heating profiles were used. Figure 15 shows the temperature profiles from two SITI tests.

Figure 16 shows the thermal diffusivity, determined from the ramp portion of the experiments. Note that for several minutes after the heater was turned on, the thermal diffusivity cannot be evaluated because the initial transients of the profile have not decayed sufficiently to produce the quadratic profile required for the analysis. After some period of time ( $\sim \mathrm{min}$ for the full density material and $\sim 8 \mathrm{~min}$ for the shredded material) the quadratic profile is established and a reasonable diffusivity can be determined. The values of 2.1 to $2.3 \times 10^{-7} \mathrm{~m}^{2} / \mathrm{s}$ for the full density material and 1.3 to $1.4 \times 10^{-7} \mathrm{~m}^{2} / \mathrm{s}$ for the shredded material were derived. These values give 1/e time constants on the order of $90 \mathrm{~s}$ and $145 \mathrm{~s}$ respectively, fitting with the expected $\sim 3$ time constants to establish the quadratic profiles. 
Figure 17 shows the heat release from SITI tests with Propellant $A$ as determined from the temperature hold portions of the experiments. Note that the baseline, full density material reached a thermal runaway state at about $90 \mathrm{~min}$, while the shredded material did not reach a thermal runaway state, even after 5 hours. That test was vented to the atmosphere; apparently enough energy escaped with the vent gases to prevent a runaway state from being reached. The propellant has a heat of explosion of $\sim 9000 \mathrm{~kJ} / \mathrm{kg}$. Interestingly, only about $3 \%$ of that energy was released prior to ignition in the full density material, whereas about $14 \%$ of the available energy was released in the shredded material.
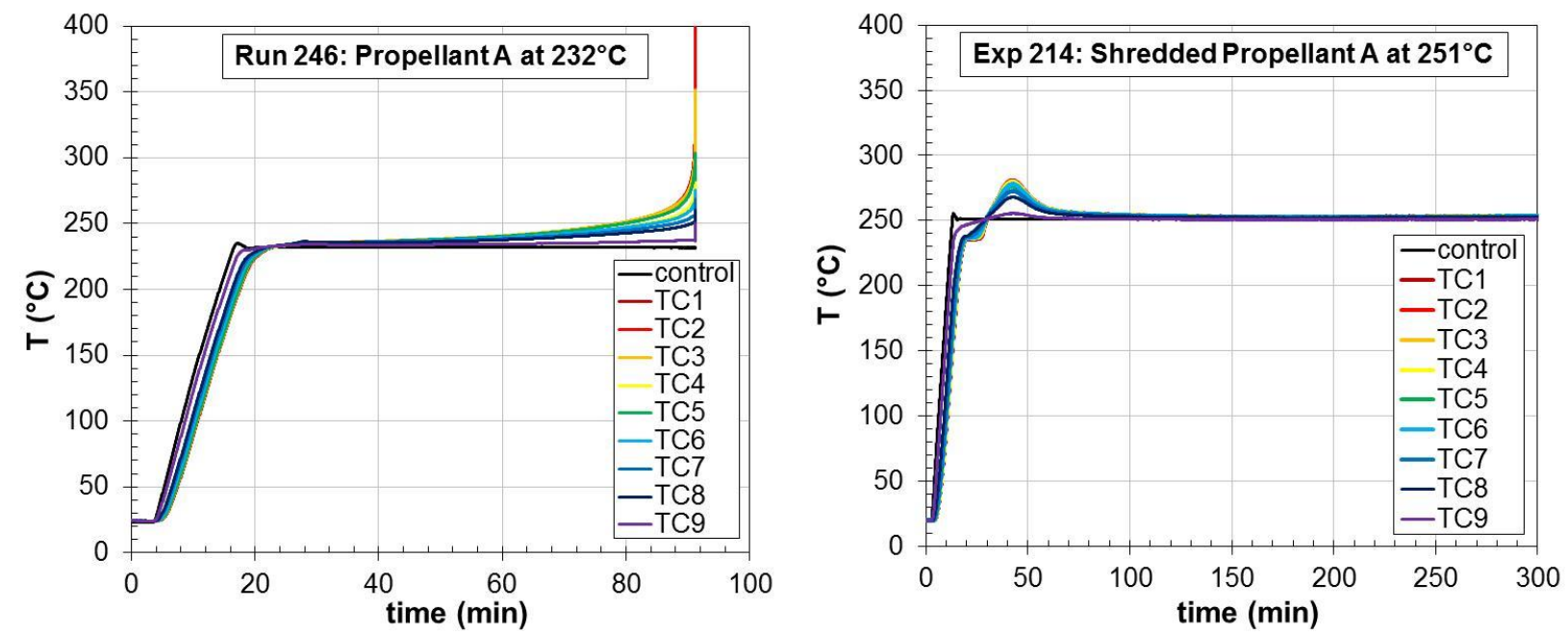

Fig. 15:Internal temperatures in SITI tests with Propellant A. Left: baseline, full density material. Right: shredded material at $52 \%$ density.
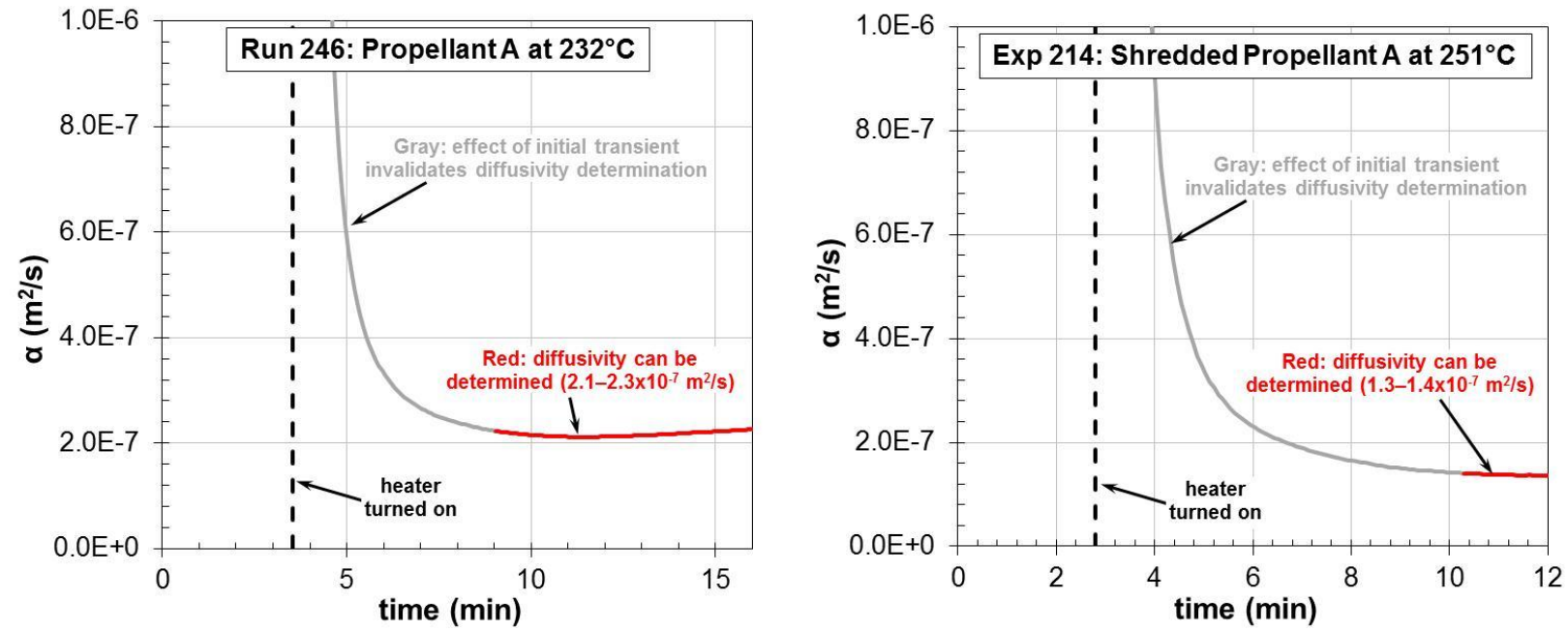

Fig. 16: Thermal diffusivity of Propellant $A$ as determined from ramp portion of SITI tests. Left: baseline, full density material. Right: shredded material at $52 \%$ density. Gray portions indicate period where initial transients invalidate results. Red portions show diffusivity. 

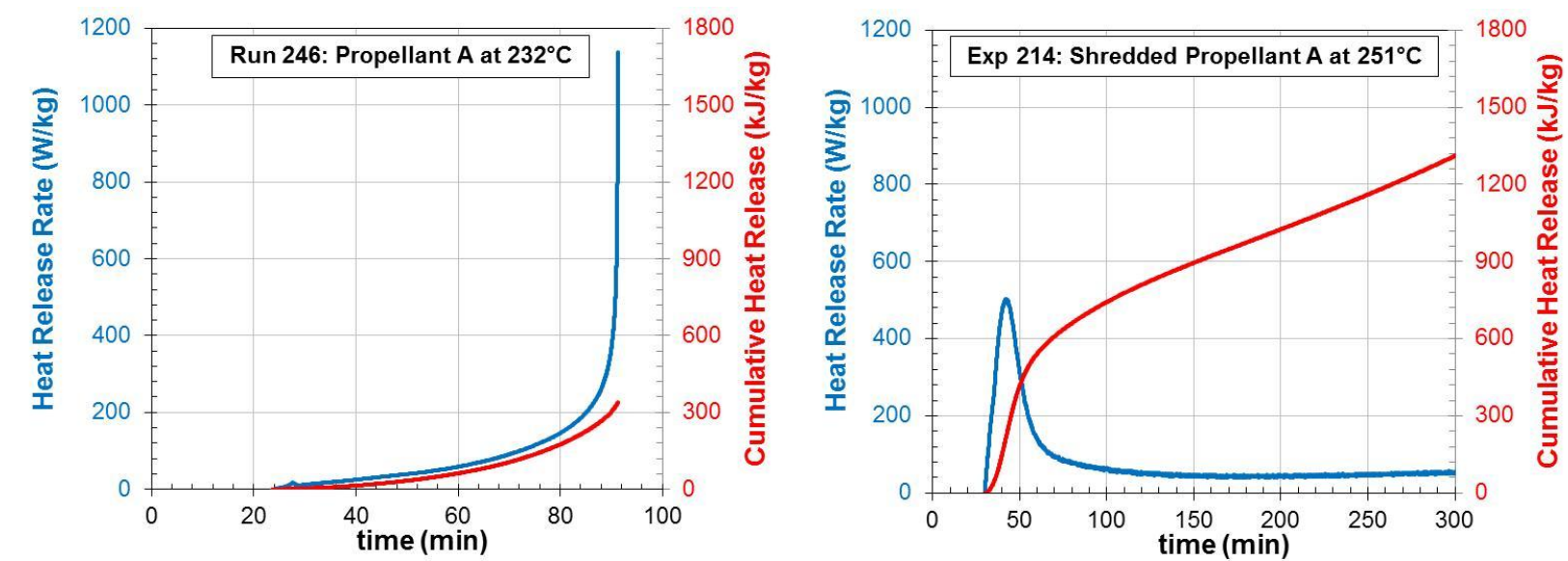

Fig. 17: Heat release from Propellant $A$ as determined from the hold portion of SITI tests. Left: baseline, full density material. Right: shredded material at $52 \%$ density. Blue curves show the instantaneous heat release, red lines show cumulative heat release during test.

\section{PBX 9502 EXPLOSIVE.}

PBX 9502 is a plastic bonded explosive made of TATB with a Kel-F polymer as a binder. Tests were conducted with two varieties of this material in SITI: full-density pressed PBX 9502 and loose, unpressed molding powder. Figure 18 shows temperature profiles for two example SITI tests with this material. On the left is the full density material tested with a $3^{\circ} \mathrm{C} / \mathrm{min}$ heating ramp. On the right is $38 \%$ dense molding powder tested with a ramp and hold at $280^{\circ} \mathrm{C}$.
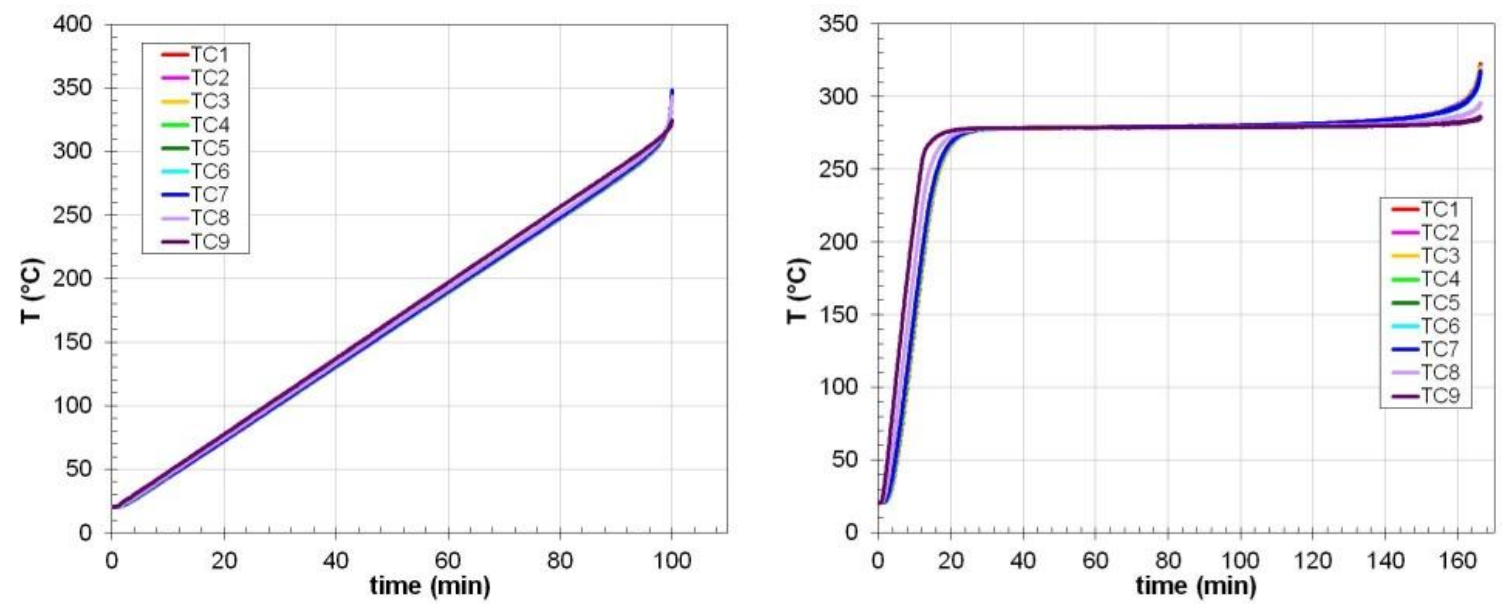

Fig. 18: Internal temperatures for SITI tests with PBX 9502. Left: full density pressed material tested with a $3^{\circ} \mathrm{C} / \mathrm{min}$ ramp. Right: $38 \%$ dense molding powder tested with a ramp and hold profile at $280^{\circ} \mathrm{C}$.

Figure 19 shows thermal diffusivity (left graph) and conductivity (right graph) derived from SITI tests with PBX 9502. Data are shown for pressed pellets at about $97 \%$ theoretical density and loose molding powder at about 38\% density. Per Table II, S-factors of 0.2268 (pellet) and 0.2247 (loose powder) were used in computing the properties for the SITI apparatus with the 0.150 " gap. Note that the pressed pellet shows a decrease in thermal conductivity with temperature over the range of $50^{\circ} \mathrm{C}$ to $270^{\circ} \mathrm{C}$. The loose molding powder does not show the same effect. It is believed that this is due to competing effects: thermal expansion increases the level of particle-to-particle contact which would lead to a higher effective conductivity counterbalanced by a decrease in the actual material conductivity. Also shown on the graph are data for thermal properties determined by experiments conducted at LLNL 
(Glascoe) and LANL (Baytos) [15, 16]. Note that our result compares very favorably, particularly with the LLNL measurements. In addition, our approach allows the data to be extended to higher temperatures.

It should be noted that there is some level of inherent variability associated with the loose powder experiments. This is to be expected because we clearly expect variations in the powder packing throughout the cavity domain. The results of seven different tests were included in Fig. 19. The min to max variability of the seven tests is about $35 \%$.

In developing this technique, we have noted sensitivities to certain parameters, notably the Sfactor. But we have not undertaken to conduct a full uncertainty analysis to determine the error bars of the data, though if this were done it is clear that uncertainties in temperature measurement will play a significant role. We note that using manufacturer's TC error limits (the larger of $\pm 2.2^{\circ} \mathrm{C}$ or $0.75 \%$ of reading) or even the special limits of error material $\left( \pm 1.1^{\circ} \mathrm{C}\right.$ or $0.4 \%$ of reading) as uncertainty bounds would result in enormous error bars for the diffusivity or conductivity. However, it is not the TC absolute accuracy which is important to this analysis technique, but rather the precision (unit-to-unit variability) which is important. We believe that our TC precision is much tighter than these absolute accuracy values, as shown by Fig. 6 . Furthermore, we have tried to reduce variability by appealing to a collection of nine TCs in the fits, rather than a single TC. Future efforts in this regard may include uncertainty analysis from TC measurements and TC placement as well as other parameters.
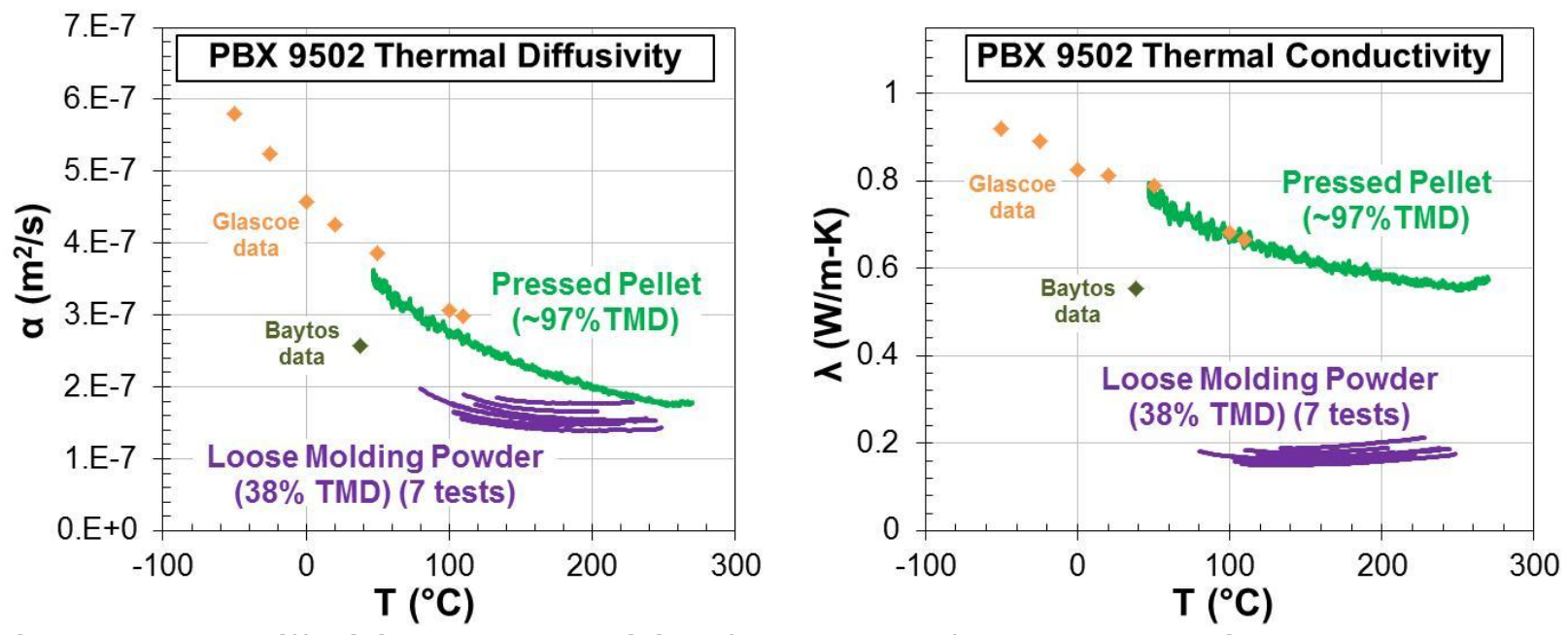

Fig. 19: Thermal diffusivity and conductivity of PBX 9502 at full pressed density and $38 \%$ dense molding powder as a function of temperature. Left: diffusivity; Right: conductivity. Also shown are experimental data from other sources $[15,16]$.

\section{CONCLUSIONS AND RECOMMENDATIONS}

In this work we have presented an approach in which temperature ramped SITI data can be used to obtain thermal diffusivity, conductivity, and energy release rates directly from experiments, by appealing to the quadratic shape of the temperature profile produced therein. Data were collected for pyrotechnic, propellant, and explosive materials; these were processed to obtain thermal properties and heat release. Comparison with available thermophysical property data for $\mathrm{KClO}_{4}$ and $\mathrm{PBX} 9502$ was made and our results are in reasonable agreement with literature values.

While the approach we have taken is not the only way to obtain thermophysical property data, it does offer some benefits. For instance, as demonstrated with several of the materials, this approach can readily accommodate powders or loose particulate materials which would be difficult to deal with in other approaches. Moreover, tests can be performed in conditions that may more closely reflect conditions in an EM containing device (at elevated temperatures and pressures, confined conditions etc.). 
The analysis techniques described here suggests potential improvements in the technique or additional work in other areas. For example, since the signal-to-noise ratio is directly proportional to heating rate (refer to Fig. 3 and Eq. 7a), it may be useful to perform tests at higher heating rates with the drawback of the first few minutes of data rendered unusable for analysis by the transient terms. Also since obtaining thermal conductivity from this approach requires knowledge of specific heat capacity, it suggests that obtaining quality data for heat capacity as a function of temperature would be of use for many materials where this data is not already known. Differential scanning calorimetry or other related techniques may be useful in this regard. Finally, while preliminary indications are favorable, it would be valuable to do a more thorough analysis to quantify the uncertainty associated with the process.

\section{ACKNOWLEDGEMENTS}

This work was supported in part by the DoD/DOE Joint Munitions program under Technical Coordination Group - III as well as internal funding at Sandia National Laboratories. The materials for testing came from a variety of sources and programs (not specifically allocated for this work). The PBX 9502 originated from Los Alamos, the Propellant A material was provided to us by Walt Gill at SNL, and the TKP output powder was manufactured by ATK. We appreciate the helpful manuscript reviews and comments by Gregg Radtke and Cole Yarrington of SNL.

\section{REFERENCES}

[1] Erikson, W. W., Hobbs, M. L., Cooper, M. A., and Oliver, M. S. "Modeling the Ignition Thresholds of Titanium Subhydride Potassium Perchlorate (THKP) Pyrotechnics," JANNAF 58th JPM, 44th CS, 32nd APS, 32nd EPSS and 26th PSHS Joint Meeting, Arlington, VA, April, 2011.

[2] http://www.astm.org/search/site-search.html?query=thermal+conductivity\#99308796

[3] Schmitt, R. G., Erikson, W. W., Kaneshige, M. J., and Renlund, A. M. "An Inverse Heat Transfer Analysis of the Sandia Instrumented Thermal Ignition Apparatus," JANNAF 39th CS, 27th APS, 21st PSHS, and 3rd MSS Joint Meeting, Colorado Springs, CO, Dec. 2003.

[4] Dodd, A. B. and Kaneshige, M. J. "Cook-off Model Development and Analysis of Energetic Materials Using Sandia Instrumented Thermal Ignition (SITI) Experimental Data," Thirteenth International Detonation Symposium, Norfolk VA, July 2006, pp. 516-526.

[5] Kaneshige, M. J., Renlund, A. M., Schmitt, R. G., and Erikson, W. W., "Cook-off Experiments for Model Validation at Sandia National Laboratories," 12th International Detonation Symposium, San Diego, CA, Aug. 2002. pp. 821-830.

[6] Cooper, M. A., Oliver, M. S., Erikson, W. W., and Hobbs, M. L., "Characterization of the Slow-Heating Behavior and Ignition Thresholds of Titanium Subhydride Potassium Perchlorate," JANNAF 58th JPM, 44th CS, 32nd APS, 32nd EPSS and 26th PSHS Joint Meeting, Arlington, VA, April, 2011

[7] Carslaw, H. S. and Jaeger, J. C. Conduction of Heat in Solids, 2nd Edition, Oxford University Press, Oxford, Great Britain, 1959, p. 104, 201, 235.

[8] Ozisik, M. N. Heat Conduction, John Wiley \& Sons, Inc., New York, NY, 1980.

[9] Cole, K. D., Beck, J. V., Haji-Sheikh, A., and Litkouhi, B., Heat Conduction Using Green's Functions, 2nd Edition, Taylor \& Francis Group, LLC, Boca Raton, FL, 2011.

[10] Chase Jr., M. W., Davies, C. A., Downey Jr., J. R., Frurip, D. J., McDonald, R. A., and Syverud, A. N. "JANAF Thermochemical Tables, Third Edition," Journal of Physical and Chemical Reference Data, Vol. 14, Supplement No. 11985.

[11]Rao, C. N. R. and Prakash, B. "Crystal Structure Transformations in Inorganic Sulfates, Phosphates, Perchlorates, and Chromates," National Bureau of Standards report NSRDS-NBS 56, Nov. 1975.

[12] Walker, D., Clark, S. M., Cranswick, L. M. D., Johnson, M. C., and Jones, R. L. "O2 Volumes at High Pressure from $\mathrm{KClO} 4$ decomposition: D" as a Siderophile Element Pump Instead of a Lid on the Core," Geochemistry Geophysics Geosystems, Vol. 3, No. 11, 1070 doi:10.1029/2001GC000225, Nov. 2002.

[13] Marmiroli, B., Manzoni, G. and Frassine, R. "Thermal Conductivity Measurement of Nanoparticle Based Solid Propellant Mixtures," Materialwissenschaft und Werkstofftechnik, V. 34, pp. 400-405, 2003. 
[14] lida, M., Matsunaga, T., Miyake, A., and Ogawa, T., "Evaluation of Effective Thermal Conductivity of Fireworks Compositions," Journal of Thermal Analysis and Calorimetry, Vol. 85, pp. 629-632, 2006.

[15] Glascoe, E. Personal Communication, Lawrence Livermore National Laboratory, 2012.

[16] Baytos, J. F., Specific Heat and Thermal Conductivity of Explosives, Mixtures, and Plastic-Bonded Explosives Determined Experimentally, Los Alamos National Laboratory, Los Alamos, NM, LA-8034MS (1979). 